\title{
Active star formation in the large Bok globule CB 34*
}

\author{
T. Khanzadyan ${ }^{1}$, M. D. Smith ${ }^{1}$, R. Gredel ${ }^{2}$, T. Stanke ${ }^{3}$, and C. J. Davis ${ }^{4}$ \\ 1 Armagh Observatory, Armagh BT61 9DG, Northern Ireland, UK \\ e-mail: mds@star.arm.ac.uk \\ 2 Centro Astronomico Hispano-Aleman, 04080 Almeria, Spain \\ e-mail: gredel@caha.es \\ 3 MPI für Radioastronomie, Auf dem Hügel 69, 53121, Bonn, Germany \\ e-mail: tstanke@mpifr-bonn.mpg.de \\ 4 Joint Astronomy Centre 660 N. A'Ohoku Place, University Park, Hilo, Hawaii 96720, USA \\ e-mail: c.davis@jach.hawaii.edu
}

Received 20 June 2001 / Accepted 29 October 2001

\begin{abstract}
We present near-infrared and millimetre observations of the large Bok globule CB 34. Two long parallel trails of $\mathrm{H}_{2}$ knots are discovered on wide-field images in the 1-0 S(1) $2.12 \mu \mathrm{m}$ emission line. These parsec scale $\mathrm{H}_{2}$ jets extend to the edge of the dark globule where they disappear without the trace of bow shocks. This suggests that the outflows physically extend into a lower density ambient medium where their terminating bows are beyond present detection limits. The two outflows are extremely well collimated and parallel to within $3^{\circ}$. The outflow mechanical luminosity, derived from CO measurements, and the shocked luminosity, estimated from the $\mathrm{H}_{2}$ emission, are similar, consistent with jet-driven non-evolving outflow structure. The jets appear to originate from the densest cores, as observed in $\mathrm{H}^{13} \mathrm{CO}^{+}$line emission. A central concentration of reddened stars and a lower density halo of less reddened stars within the globule are revealed by $J H K$ photometry. Disordered motions are observed in the CO $J=2-1$ line velocity channel maps and can be driven by the power of the outflows emanating from dense cores. We sketch a picture for the star formation history of the globule in which two star phases have been formed. A weak diffuse emission halo is detected in the near infrared with colours consistent with either scattered light or a ro-vibrational $\mathrm{H}_{2}$ cascade. We propose that the halo is produced by ongoing $\mathrm{H}_{2}$ formation. Cloud evolution and halo $\mathrm{H}_{2}$ formation timescales are then both a few $\times 10^{5} \mathrm{yr}$. Thus, we may be witnessing the formation of a molecular cloud out of diffuse atomic gas. This supports a scheme in which this Bok globule has formed independently rather than through dislocation from a nearby molecular cloud.
\end{abstract}

Key words. infrared: ISM - stars: formation - ISM: jets and outflows - ISM: clouds

\section{Introduction}

Bok globules are isolated clouds of dense molecular gas (Bok \& Reilly 1947). They are recognised in the optical as sharply outlined dark patches against a background of stars. Bok \& Reilly's (1947) statement that the globules were probably sites where stars would form has been confirmed (e.g., Yun \& Clemens 1990). We have begun a project to study Bok globules, to determine how they

Send offprint requests to: T. Khanzadyan,

e-mail: tig@star.arm.ac.uk

* Based on observations collected at the German-Spanish Astronomical Centre, Calar Alto, operated by the Max-PlanckInstitut für Astronomie, Heidelberg, jointly with the Spanish National Commission for Astronomy. Includes observations made at the $30 \mathrm{~m}$ IRAM telescope (Pico Veleta, Spain). form, evolve and disperse. Their significance lies not only in their illustration of a distinct mechanism leading to stars but also that a considerable proportion of gas in the Galaxy is processed through them. A total of $10^{10} M_{\odot}$ is processed in the lifetime of the Galaxy, given a globule lifetime of $10^{6}$ years (Launhardt \& Henning 1997), a number of $10^{5}$ (Clemens et al. 1991), along with a mass of $10 M_{\odot}$. Hence, many field stars including our Sun, as well as the enigmatic isolated T Tauri stars (Yun et al. 1997; Launhardt \& Henning 1997), could find their origin in Bok globules.

Globules are not a homogeneous group of objects. They span a range in masses from $\sim 1-100 M_{\odot}$ and their angular sizes are typically a few arcminutes which corresponds to sizes of $0.1-1$ parsec, according to their distance. Therefore, a single low-mass star could form or a small 
group including high-mass stars could result. Moreover, we cannot expect to uncover a single evolutionary scheme for all Bok globules, if any scheme exists. Bok globules could (1) form spontaneously from small growing perturbations, (2) be triggered by a large pressure wave or (3) evolve after fragmenting from a larger molecular cloud. They are not perfectly isolated. Usually, some connection with a distant molecular cloud complex is found.

We present here near-infrared and millimetre data for the Bok globule CB 34 (Clemens \& Barvanis 1988), in order to determine its evolutionary stage. CB 34 belongs to a class of distant massive globules. We thus wish to examine if a few powerful outflows, corresponding to those expected from high-mass stars, are being generated. The Gemini OB1 cloud is $\approx 2^{\circ}$ east of CB 34 at an estimated distance of $1.5 \mathrm{kpc}$ from us. Supposing that the two formed at the same time, with the early CB 34 being stripped off from the main outskirts of the cloud and separating at $10 \mathrm{~km} \mathrm{~s}^{-1}$, would then yield an age of at least $5 \times 10^{6} \mathrm{yr}$. This is uncomfortably old, being equal to the average age for globule dispersal derived by Launhardt \& Henning (1997).

CB 34 is certainly in an advanced stage. It has successfully formed dense molecular clumps and protostars (Launhardt \& Henning 1997; Huard et al. 2000) as well as being associated with young stars (Alves et al. 1997). The chemical age of the globule exceeds $10^{5} \mathrm{yr}$ (Scappini et al. 1998) (although this result is sensitive to the assumed density of $10^{4} \mathrm{~cm}^{-3}$ ). Collimated outflows also testify to active star formation. A bipolar CO outflow from cool gas (Yun \& Clemens 1994), shocked optical knots of atomic emission lines from radiative shocks (Herbig-Haro objects), as well as an infrared outflow from vibrationallyexcited molecular hydrogen (Yun \& Clemens 1994) have been reported. Protostellar outflows can be several parsecs long. Therefore, to search for evidence for evolved outflows, we chose CB 34 for wide-field infrared observations in the $2.12 \mu \mathrm{m} \mathrm{H} \mathrm{H}_{2}$ line.

We also present $J H K$ photometry for CB 34 and compare it to previous results of Moreira \& Yun (1995). We discuss the depth in the globule and the protostellar stage of individual stars. Finally, we have obtained velocitychannel maps in $\mathrm{CO}, \mathrm{H}^{13} \mathrm{CO}^{+}$and $\mathrm{SiO}$, in order to determine the location of the densest cores and to search for further outflows.

\section{Observations and data reduction}

\subsection{Near-infrared observations}

The near infrared observations of CB 34 were carried out during the nights of December 8-9, 2000 using Omega Prime (Bizenberger et al. 1998) at the Calar Alto $3.5 \mathrm{~m}$ telescope. Omega Prime is equipped with a Rockwell $1024 \times 1024$ pixel HAWAII array detector. It provides a pixel scale of $0.4 \mathrm{arcsec} /$ pixel scale and a total field of view of $6.8 \times 6.8 \operatorname{arcmin}^{2}$ on the sky.
Images were obtained in the $J, H$ and $K_{\mathrm{s}}$ broadband filters, with filter passbands centered at $\lambda=1.275,1.645$, and $2.196 \mu \mathrm{m}$, respectively, as well as in the narrowband $\mathrm{H}_{2} 1-0 \mathrm{~S}(1)$ filter centered at $\lambda=2.125 \mu \mathrm{m}$ and a width of $0.0206 \mu \mathrm{m}$. The detector integration time (DIT) was $1.677 \mathrm{~s}$, and the total sum per "frame" of $47 \times 1.677 \mathrm{~s}$ for all four filters was achieved by using the new Full MPIA read mode (Bizenberger et al. 1998). The total integration times were $1500 \mathrm{~s}$ in $J$ and $H, 2000 \mathrm{~s}$ in $K_{\mathrm{s}}$ and finally $3800 \mathrm{~s}$ in the $\mathrm{H}_{2}$ narrow band.

Images were obtained by positioning the globule in the center and in each of the four quadrants of the detector. The method avoids the explicit acquisition of sky frames and maximises the time spent on the object - see Cowie et al. (1990) and Stanke (2000) for a detailed description. Difference images obtained on a flat field screen with and without illumination by a halogen lamp were used to define the pixel-to-pixel response pattern of the detector. We identified hot and cold pixels using the flatfield frames taken each night. The bad pixels were flagged and excluded from processing during the following steps of data reduction.

Individual frames in each filter were passed through two stages of the DIMSUM ${ }^{1}$ package: First Pass and Mask Pass. During the First Pass sky subtraction was performed automatically by median filtering and object rejection using a $5 \sigma$ iterated rejection mechanism (Eisenhardt et al. 1996). This functions very well under the above observational strategy. Then, by applying a threshold algorithm to the ratio of the image and median filtered image, a cosmic ray mask was created and utilised. In the Mask Pass, the images were mosaiced and a wide field image obtained by choosing the reference star on the central frame and shifting and orientating the frames towards it. The thresholding for background detection and object masking were performed for the mosaiced picture.

Final mosaics of $8.8 \times 8.8 \operatorname{arcmin}^{2}$ are obtained after the 2 runs. The results for $J, H, K_{\mathrm{s}}$ and $\mathrm{H}_{2}$ are displayed in Figs. $14 \mathrm{a}-\mathrm{d}$, respectively.

The astrometric calibration was performed by use of stars from the Hubble Space Telescope guide star catalog (GSC) and the SIMBAD ${ }^{2}$ data base which were identified on our mosaics. We compare our images with those from $2 \mathrm{MASS}^{3}$. There is a good positional accuracy of the order of $1^{\prime \prime}$, which is similar to the positional errors in the SIMBAD and GSC data bases.

In the final stage of data reduction, the $\mathrm{H}_{2}$ image was continuum subtracted. We took $\mathrm{H}_{2}(s u b)=\left(\mathrm{H}_{2} \times C\right)$ - $K_{\mathrm{s}}$. Where the constant $C$ was determined from the width of the $K_{\mathrm{S}}$ and $\mathrm{H}_{2}$ filters and by eye examination of

\footnotetext{
${ }^{1}$ DIMSUM: Deep Infrared Mosaicing Software, Um ... IRAF external package developed by Eisenhardt et al. (1996).

${ }^{2}$ SIMBAD is online database, operated at CDS, Strasbourg, France.

3 2MASS two micron all sky survey project is collaboration between The University of Massachusetts and the Infrared Processing and Analysis Center (JPL/Caltech).
} 


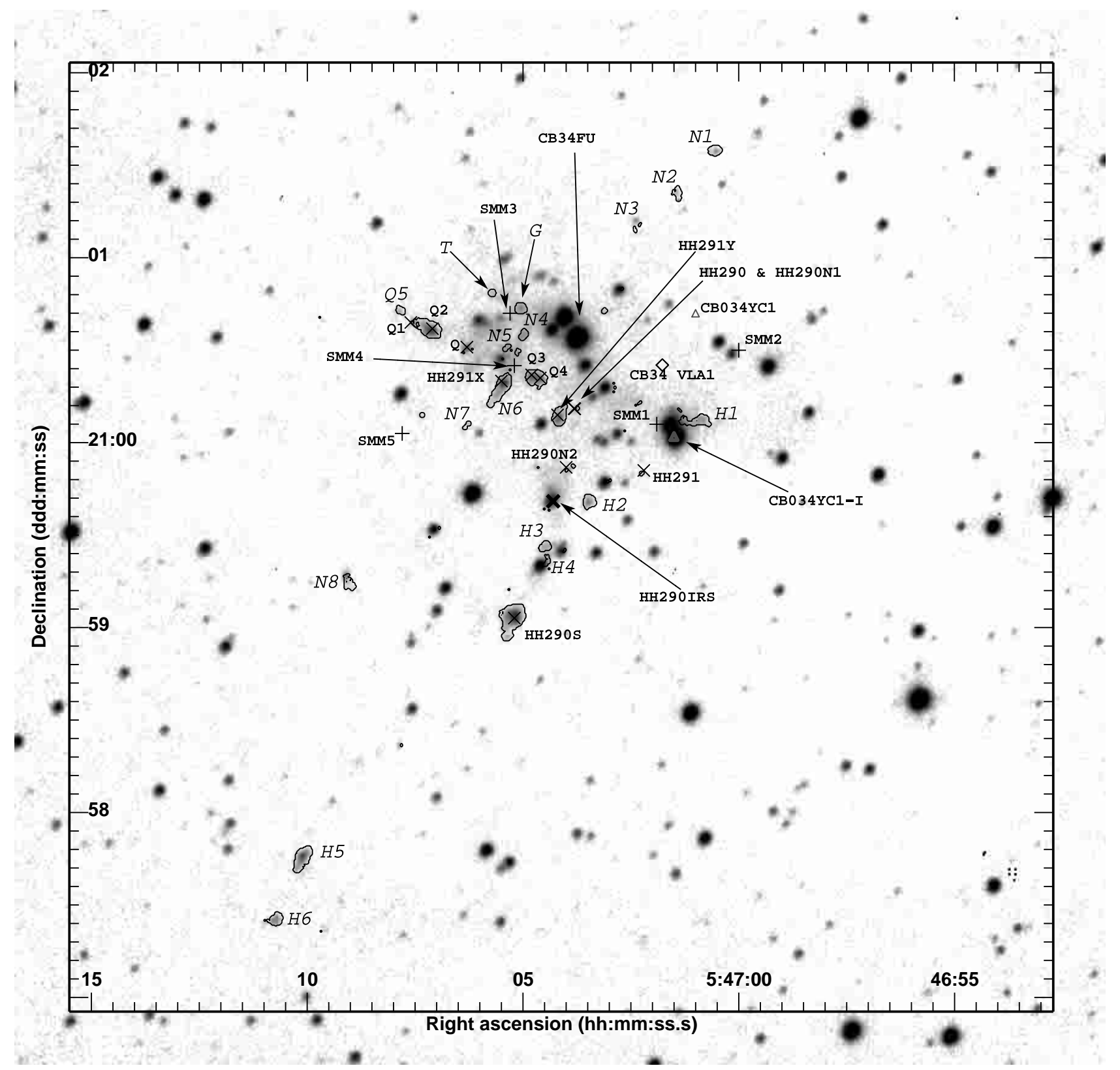

Fig. 1. The central part of CB 34 in $\mathrm{H}_{2}$. Total integration of this frame is $\approx 3800 \mathrm{~s}$. Crosses "+" are SMM objects from Huard et al. (2000) and the " $\times$ " are $\mathrm{HH}$ objects and $\mathrm{H}_{2}$ knots discovered by Moreira \& Yun (1995). CB34 VLA1 is marked by a "diamond" (Yun et al. 1996). The YSOs NIR counterparts CB034YC1 and CB034YC1-I ("triangles") are from Yun \& Clemens (1994). Our discovered objects are indicated as chains of $\mathrm{H}$ and $\mathrm{N}$ letters as well as $\mathrm{G}$ and $\mathrm{T}$. One more Q knot has been found - Q5.

the subtracted image (in order to subtract the majority of field stars). From the subtracted image, $\mathrm{H}_{2}$ excitation knots were revealed. Finally, an $\mathrm{H}_{2}$ excitation mask was made (defined as 1 where $\mathrm{H}_{2}$ excitation exist and 0 where not) which was multipled by the actual $\mathrm{H}_{2}$ image in order to overlay it on the unsubtracted $\mathrm{H}_{2}$ image. This distinguishes the $\mathrm{H}_{2}$ excitation objects in Fig. 1 .

\section{2. $\mathrm{H}^{13} \mathrm{CO}^{+}$and ${ }^{12} \mathrm{CO}(2-1)$ observations}

We obtained millimetre line maps in several molecular tracers of the central regions of CB34 using the
IRAM 30 m telescope on Pico Veleta, Spain, on April 28th, 29 th and 30 th of 2001 .

The observations were done using 4 receivers simultaneously, with the backends configured to observe the following lines: the ${ }^{12} \mathrm{CO}(2-1)$ line (at $230.538 \mathrm{GHz}$ ) with the A230 receiver and the autocorrelator as backend with a frequency resolution of $320 \mathrm{kHz}$ (velocity resolution of $0.42 \mathrm{~km} \mathrm{~s}^{-1}$ ); the ${ }^{13} \mathrm{CO}(2-1)$ line (at $220.399 \mathrm{GHz}$ ) with the B230 receiver and the autocorrelator (same resolution as for the ${ }^{12} \mathrm{CO}(2-1)$ line); the ${ }^{12} \mathrm{CO}(1-0)$ line (at $115.271 \mathrm{GHz}$ ) with the $\mathrm{B} 100$ receiver and the autocorrelator (frequency resolution $320 \mathrm{kHz}$ corresponding to 
$0.83 \mathrm{~km} \mathrm{~s}^{-1}$ ); the $\mathrm{SiO}(2-1 v=0)$ line (at $86.847 \mathrm{GHz}$ ) with the A100 receiver and the $1 \mathrm{MHz}$ backend (velocity resolution $3.5 \mathrm{~km} \mathrm{~s}^{-1}$ ); and finally the $\mathrm{H}^{13} \mathrm{CO}^{+}(1-0)$ line (at $86.754 \mathrm{GHz}$ ) also with the A100 receiver and the $100 \mathrm{kHz}$ backend (velocity resolution $0.35 \mathrm{~km} \mathrm{~s}^{-1}$ ).

The final maps consist of a number of smaller raster maps. Of those, the first map centred roughly on the north-eastern core containing SMM4 was taken on a $4^{\prime \prime}$ grid covering $40^{\prime \prime} \times 40^{\prime \prime}$ (i.e., fully sampled even for the high-frequency $\mathrm{CO}(2-1)$ lines; the beamsize at $230 \mathrm{GHz}$ is about $11^{\prime \prime}$ ), the remaining maps were taken on $8^{\prime \prime}$ or $10^{\prime \prime}$ grids, i.e., fully sampled for the low-frequency data (the beamsize is about $27^{\prime \prime}$ at $86 \mathrm{GHz}$ and $22^{\prime \prime}$ at $115 \mathrm{GHz}$ ), but undersampling the high-frequency data.

The data were flux calibrated using the standard hot/cold load calibration procedure at the $30 \mathrm{~m}$. However, it should be noted that part of the data were taken in cloudy or foggy weather, thus the calibration (particularly for the high frequency $\mathrm{CO}(2-1)$ data) might be severely inaccurate for some of the data. We will therefore only use general morphological information rather than interpret the brightness of the features found in the data.

We present here only velocity channel maps of the ${ }^{12} \mathrm{CO}(2-1)$ line and the $\mathrm{H}^{13} \mathrm{CO}^{+}$line. No emission was detected in the $\mathrm{SiO}$ line (a shock tracer, e.g., Schilke et al. 1997); apparently the shocks in CB 34 are not strong enough and too far away to produce detectable $\mathrm{SiO}$ emission.

\section{Results}

\subsection{Newly discovered objects}

The central part of CB 34 in the continuum subtracted $(1,0) \mathrm{S}(1)$ line of molecular hydrogen is shown in Fig. 1. The lowest $\mathrm{H}_{2}$ contours are two standard deviations above the noise in the sky level. The marked objects are taken from Huard et al. (2000; crosses "+"), Moreira \& Yun (1995; "×"s), Yun et al. (1996; "diamonds") and Yun \& Clemens (1994; "triangles") which are overlaid using the SIMBAD online data base.

Newly found $\mathrm{H}_{2}$ objects are marked with the letters H1-H6, N1-N6, G, T and Q5. The objects Q1-Q4 are $\mathrm{H}_{2}$ emission objects discovered by Moreira \& Yun (1995). Our knot Q5 appears to be associated with Q1-Q4.

An estimate of the surface brightness averaged over a $2^{\prime \prime}$ aperture of the newly discovered emission objects is given in Col. 4 of Table 1. Columns 2 and 3 of Table 1 contain the right ascension and declination of the objects.

\subsection{Infrared photometry}

The sky conditions during the observations were less than optimal and non-photometric in particular, which does not allow us to derive independent absolute photometry. Here, we estimate fluxes of the emission objects in CB 34 from a
Table 1. New object positions and fluxes.

\begin{tabular}{|c|c|c|c|}
\hline Object & $\begin{array}{c}\text { RA } \\
(2000.0)\end{array}$ & $\begin{array}{c}\text { Dec } \\
(2000.0)\end{array}$ & $\begin{array}{c}\mathrm{H}_{2} v=1-0 \mathrm{~S}(1)\left(\times 10^{-18}\right) \\
\left(\mathrm{Wm}^{-2} \operatorname{arcsec}^{-2}\right)\end{array}$ \\
\hline H1 & $05^{\mathrm{h}} 47^{\mathrm{m}} 0059$ & $21^{\circ} 00^{\prime} 08^{\prime \prime}$ & 2.45 \\
\hline $\mathrm{H} 2$ & $05^{\mathrm{h}} 47^{\mathrm{m}} 03^{\mathrm{s}} .5$ & $20^{\circ} 59^{\prime} 41^{\prime \prime}$ & 3.29 \\
\hline H3 & $05^{\mathrm{h}} 47^{\mathrm{m}} 04^{\mathrm{s}} .5$ & $20^{\circ} 59^{\prime} 26^{\prime \prime}$ & 1.85 \\
\hline $\mathrm{H} 4$ & $05^{\mathrm{h}} 47^{\mathrm{m}} 04 \mathrm{~s} .4$ & $20^{\circ} 59^{\prime} 23^{\prime \prime}$ & 1.56 \\
\hline H5 & $05^{\mathrm{h}} 47^{\mathrm{m}} 10^{\mathrm{s}} .1$ & $20^{\circ} 57^{\prime} 46^{\prime \prime}$ & 6.93 \\
\hline H6 & $05^{\mathrm{h}} 47^{\mathrm{m}} 10^{\mathrm{s}} .7$ & $20^{\circ} 57^{\prime} 25^{\prime \prime}$ & 2.86 \\
\hline N1 & $05^{\mathrm{h}} 47^{\mathrm{m}} 00^{\mathrm{s}} \cdot 5$ & $21^{\circ} 01^{\prime} 35^{\prime \prime}$ & 1.92 \\
\hline N2 & $05^{\mathrm{h}} 47^{\mathrm{m}} 01^{\mathrm{s}} .4$ & $21^{\circ} 01^{\prime} 21^{\prime \prime}$ & 1.21 \\
\hline N3 & $05^{\mathrm{h}} 47^{\mathrm{m}} 02^{\mathrm{s}} .4$ & $21^{\circ} 01^{\prime} 12^{\prime \prime}$ & 1.11 \\
\hline N4 & $05^{\mathrm{h}} 47^{\mathrm{m}} 05^{\mathrm{s}} .0$ & $21^{\circ} 00^{\prime} 35^{\prime \prime}$ & 2.84 \\
\hline N5 & $05^{\mathrm{h}} 47^{\mathrm{m}} 05^{\mathrm{s}} \cdot 2$ & $21^{\circ} 00^{\prime} 30^{\prime \prime}$ & 2.08 \\
\hline N6 & $05^{\mathrm{h}} 47^{\mathrm{m}} 05^{\mathrm{s}} 6$ & $21^{\circ} 00^{\prime} 16^{\prime \prime}$ & 3.06 \\
\hline N7 & $05^{\mathrm{h}} 47^{\mathrm{m}} 06^{\mathrm{s}} 3$ & $21^{\circ} 00^{\prime} 06^{\prime \prime}$ & 0.76 \\
\hline N8 & $05^{\mathrm{h}} 47^{\mathrm{m}} 09.1$ & $20^{\circ} 59^{\prime} 17^{\prime \prime}$ & 3.58 \\
\hline G & $05^{\mathrm{h}} 47^{\mathrm{m}} 05^{\mathrm{s}} \cdot 1$ & $21^{\circ} 00^{\prime} 44^{\prime \prime}$ & 3.78 \\
\hline $\mathrm{T}$ & $05^{\mathrm{h}} 47^{\mathrm{m}} 05^{\mathrm{s}} .7$ & $21^{\circ} 00^{\prime} 49^{\prime \prime}$ & 0.85 \\
\hline Q5 & $05^{\mathrm{h}} 47^{\mathrm{m}} 07^{\mathrm{s}} .9$ & $21^{\circ} 00^{\prime} 43^{\prime \prime}$ & 1.69 \\
\hline
\end{tabular}

relative photometry obtained from a comparison with the same knots which were found by Alves \& Yun (1995).

For the $J H K_{\mathrm{s}}$ photometry, we use the IRAF DAOPHOT routine (Stetson 1987) to identify stars which are simultaneously present in the $J, H$, and $K_{\mathrm{s}}$ frames and to obtain instrumental stellar magnitudes. Then, instrumental magnitudes were compared with the same objects' absolute magnitudes obtained from the work of Alves \& Yun (1995) in order to detect magnitude transformation constants. Then, using the same constants for all our detected stars, the instrumental magnitudes were transformed to absolute magnitudes. This transformation method yields errors of no more than $15 \%$ in comparison with the results from Alves \& Yun (1995), although in some cases the values are identical (cf. objects A, C and D).

Figure 2 identifies the 14 most reddened objects found in this way, labelled with the same letters $\mathrm{A}-\mathrm{S}$ as used by Alves \& Yun (1995). These objects are represented by filled circles in the $(J-H)$ and $(H-K)$ colour-colour diagrams of Fig. 3. The solid line in the figure represents the location of the un-reddened main-sequence stars (Koornneef 1983). The two parallel dashed lines are the reddening vectors which define the reddening band for normal stellar photospheres. Objects with colours that fall outside and to the right of this band are sources with intrinsic infrared excess emission (Lada \& Adams 1992).

Figure 4 shows the spatial distribution of all sources detected simultaneously in the $J, H$ and $K_{\mathrm{s}}$ images. Those with $(H-K)>1.0$ are represented by filled circles in Figs. 2, 4 and 5. Crosses mark objects with $(H-K) \leq$ 1.0. From Figs. 4 and 5 one can derive a core radius of the Bok globule of approximately $50^{\prime \prime}-60^{\prime \prime}$ which contains all those objects with $(H-K)>1.6$. 


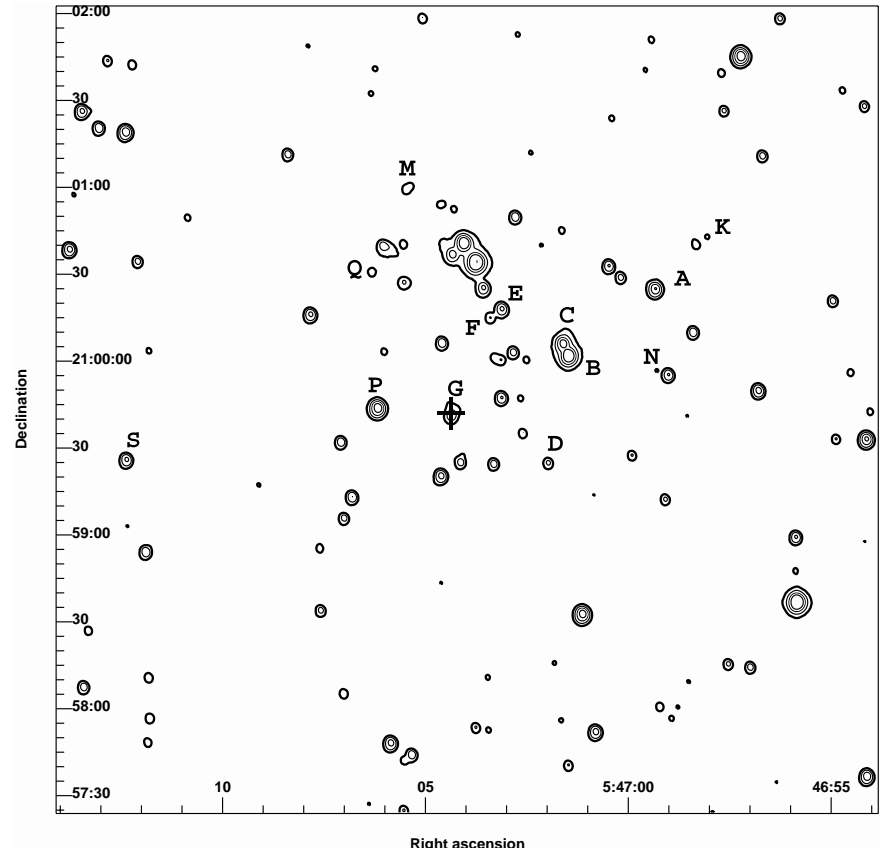

Fig. 2. Isophotes of the central region of CB 34 in $K_{\mathrm{s}}$ covering an area of about $2.5 \times 2.5 \mathrm{arcmin}^{2}$. East is left, north is up. Letters A, B, C, D, E, F, G, K, L, M, N,P,Q and S are the 14 reddest objects. Notice Object $\mathrm{S}$ which has large $[\mathrm{H}-$ $\mathrm{K}$ ] and is located at the edge of the cloud, suggesting that it is a background star.

A number of objects with large $(H-K)$ are located at the edge of the cloud (cf. Fig. 3 in comparison with Figs. 2, 4 and 5). Those objects (cf. $S, N$ and $K$ ) are probably background stars. Because of the large distance of $1500 \mathrm{pc}$ to the globule (Carpenter et al. 1995), 1-2 of the red stars located within the globule may be red foreground objects and not embedded. Objects $\mathrm{C}$ and $\mathrm{G}$ are located in the region of the $[(J-H)$ vs. $(H-K)]$-plane which has been explained by disk emission (Strom et al. 1989) and are class II objects (Adams et al. 1987). Objects $\mathrm{A}, \mathrm{B}, \mathrm{D}, \mathrm{E}$, and $\mathrm{L}$ have large $(H-K)$ and are located in the region of the diagram characterized by large extinction corresponding mostly to class I sources (Lada \& Adams 1992).

The near-infrared colours of the objects A, B, C, D, E, G and L give us confidence that they are young stellar objects (YSOs), surrounded by varying amounts of circumstellar material, and that they formed recently as a part of ongoing star formation in the CB 34 Bok globule. This is in good agreement with results from Alves \& Yun (1995).

\section{Discussion}

\subsection{The outflows}

We identify three powerful near-infrared $\mathrm{H}_{2}$ outflows which we will call " $\mathrm{H}_{2}$ jets", as shown in Fig. 6. These are designated the $\mathrm{H}, \mathrm{N}$ and $\mathrm{Q}$ jets. The $\mathrm{Q} \mathrm{H}_{2}$ jet has been previously reported in the NIR by Moreira \& Yun (1995).

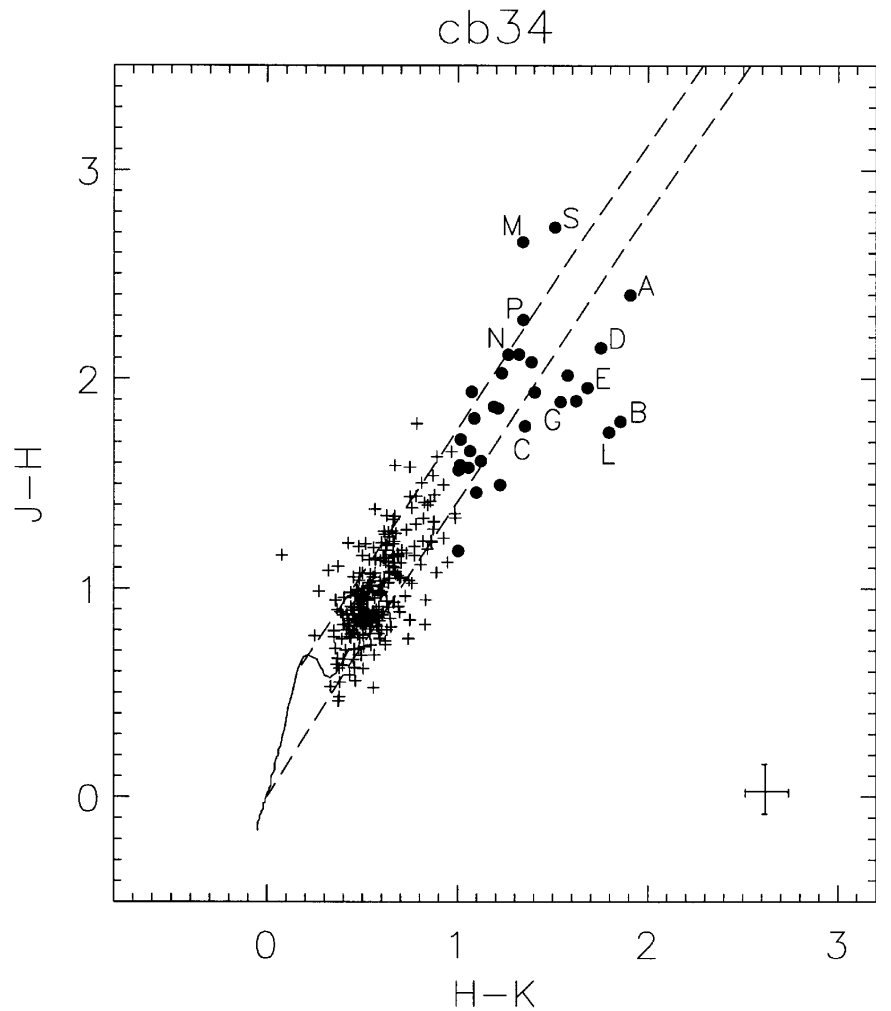

Fig. 3. Near-infrared colour-colour diagram for all sources detected in the $J, H$ and $K_{\mathrm{s}}$ bands simultaneously. The solid line is the location of the un-reddened main-sequence stars (Koornneef 1983). Dashed lines are two parallels to the reddening vector which define the reddening band for normal stellar photospheres. Objects with $(H-K)>1.0$ are marked with filled circles (see Figs. 2, 4 and 5). Crosses are objects with $(H-K) \leq 1.0$. Letters represent the same objects as in Fig. 2. The size of the error bars is shown in the lower right-hand corner.

Note the proximity of optical $\mathrm{HH}$ objects to the $\mathrm{H}$ jet. Note also that there are several knots of $\mathrm{H}_{2}$ emission not directly identified with these jets (e.g. T and G Fig. 15) which may belong to further jets. Here, however, we remain conservative, requiring the presence of at least four aligned knots before we declare an $\mathrm{H}_{2}$ jet.

The $2.12 \mu \mathrm{m}$ emission line is from the 1-0 S(1) transition of molecular hydrogen. This arises from vibrationally excited $\mathrm{H}_{2}$, probably with a temperature in the range $1000-3000 \mathrm{~K}$, excited in the remote locations by shock waves. No local source of UV radiation to suggest fluorescent excitation is present near the majority of $\mathrm{H}_{2}$ knots. The shocks are being driven, however, from distant protostars through jets or accumulated gas in bullets.

The $\mathrm{H}$ and $\mathrm{N}$ jets have required wide-field cameras to be revealed. They alter our view of the evolution of the Bok globule. These two jets are considerably longer than the Q jet. We assume here a distance to CB 34 of $1500 \mathrm{pc}$ (Carpenter et al. 1995) to yield projected sizes of $1.59 \mathrm{pc}\left(219^{\prime \prime}\right)$ for H1-H6, $1.39 \mathrm{pc}\left(191^{\prime \prime}\right) \mathrm{N} 1-\mathrm{N} 8$ and $1.17 \mathrm{pc}\left(140^{\prime \prime}\right)$ Q5-Q4-HH290N1. We find no evidence for further extensions to these jets out to a scale of $5^{\prime}$. 


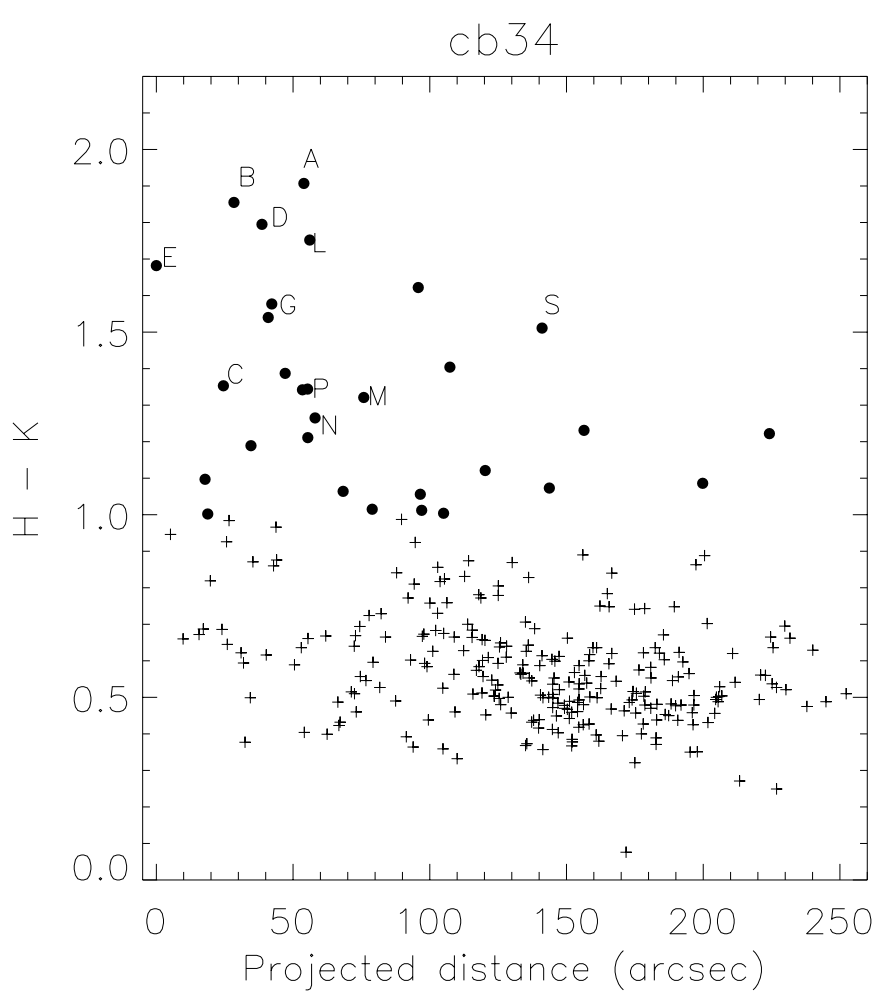

Fig. 4. Plot of the $[H-K]$ colour as a function of projected angular distance to an estimated centre point object-E, which was chosen by visual examination of the cloud (cf. Figs. 1 and 2). The symbols are the same as described in Fig. 3.

Supposing a total expansion speed of $100 \mathrm{~km} \mathrm{~s}^{-1}$ yields dynamical ages of just $1.2-1.4 \times 10^{4} \mathrm{yr}$. This would indicate that the driving protostars are in the late Class 0 regime. This age is misleading, however, since the outer parts of the outflows decelerate in the Class 1 stage. Class 1 near infrared outflows are not significantly longer than Class 0 outflows (Stanke 2000), consistent with models which account for outflow deceleration (Smith 2000).

One of the most striking features is the high collimation of the $\mathrm{H}_{2}$ jets. Angles subtended by individual components, as well as the overall collimation, of the $\mathrm{H}$ and $\mathrm{N}$ jets are less than three degrees. We define a collimation angle which is independent of the location of the driving source. This is: collimation angle $=$ arc$\tan$ (maximum transverse extent/total linear extent). This yields collimation angles of $2.7^{\circ}$ (H-flow) and $2.3^{\circ}(\mathrm{N}$ flow). Recent three dimensional simulations of hydrodynamic molecular jets show very high collimation, with jet material being focussed while ambient gas is swept aside (Rosen \& Smith 2001).

The energy of the CO $J=1-0$ outflow, scaled to the distance of $1500 \mathrm{pc}$, is $1.8 \times 10^{45} \mathrm{erg}$ (Yun \& Clemens 1994) and the CO momentum is $26.3 M_{\odot} \mathrm{kms}^{-1}$. Yun \& Clemens (1994) derived a mechanical luminosity $L_{\text {mech }}=$ $0.37 L_{\odot}$ and a dynamical age of just $3.8 \times 10^{4}$ years for the CO. This, however, assumed the existence of a high velocity wind rather than the measured $\mathrm{CO}$ speed of $\sim 5 \mathrm{~km} \mathrm{~s}^{-1}$. The latter speed, as usually assumed to calculate $\mathrm{CO}$

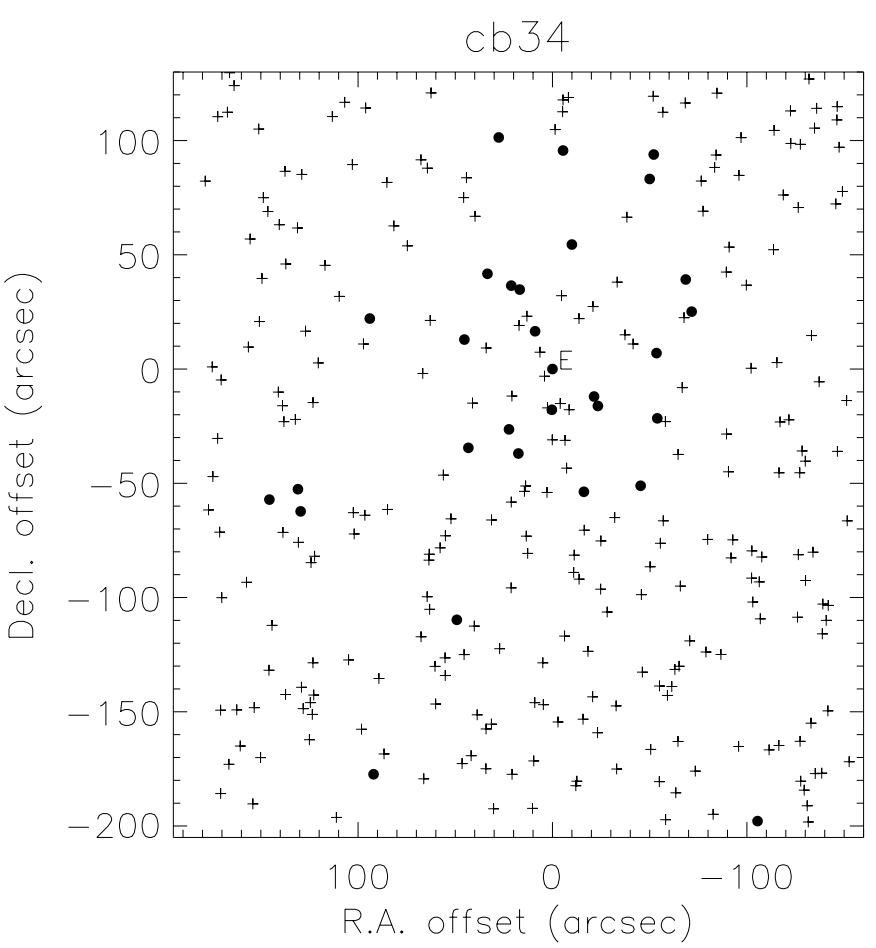

Fig. 5. Spatial distribution of all the sources seen in the 3 bands simultaneously. The offset centre is the object E.

dynamical ages, is confirmed here in our CO $J=2-1$ velocity channel maps (see below). The dynamical age is then $7.6 \times 10^{5}$ years. The mechanical luminosity is reduced to $L_{\text {mech }}=0.019 L_{\odot}$ and the momentum flow rate becomes $F_{\mathrm{CO}}=3.5 \times 10^{-5} M_{\odot} \mathrm{kms}^{-1} \mathrm{yr}^{-1}$. This is a moderately high momentum flux, and would typically be driven from a Class 1 protostar of bolometric luminosity $10 L_{\odot}$ or a Class 0 protostar of luminosity $1 L_{\odot}($ e.g. Smith 2000). The submillimetre cores could certainly contain either object, since their bolometric luminosities are $40 L_{\odot}$ (SMM1) and $20 L_{\odot}(\mathrm{SMM} 4)$.

The total luminosities in the $\mathrm{H}_{2} 1-0 \mathrm{~S}(1)$ line are $2.2 \times 10^{-4} L_{\odot}(\mathrm{H}$ flow $)$ and $1.9 \times 10^{-4} L_{\odot}(\mathrm{N}$ flow $)$, on summing up the individual knot luminosities. Applying factors based on general models, these values convert to total shocked $\mathrm{H}_{2}$ radiation of $2-5 \times 10^{-3} L_{\odot}$ per outflow, for typical C-type or J-type shocks (Smith 1995). We then estimate that the total shock power from all molecular and neutral atomic species $\left(\mathrm{CO}, \mathrm{H}_{2} \mathrm{O}, \mathrm{O}[\mathrm{I}]\right.$, etc.) is $\sim 2-$ $5 \times 10^{-2} L_{\odot}$ per outflow (Smith 1991). We assume here that the outflows are predominantly located on the edges of the globule where there will be no significant infrared extinction. Thus, the mechanical and shocked luminosities are comparable given an outflow age of $7.6 \times 10^{5} \mathrm{yr}$. Such a relationship is expected when an outflow is jet-driven for long extended periods. At this age, however, we could expect that the outflow has had time to amass a reservoir of $\mathrm{CO}$ gas whereas only feeble shocked $\mathrm{H}_{2}$ gas would be detected as the jets' power diminishes. Hence, we suggest that the present shocked $\mathrm{H}_{2}$ is being generated from a younger outflow while previous bipolar outflows dominate 


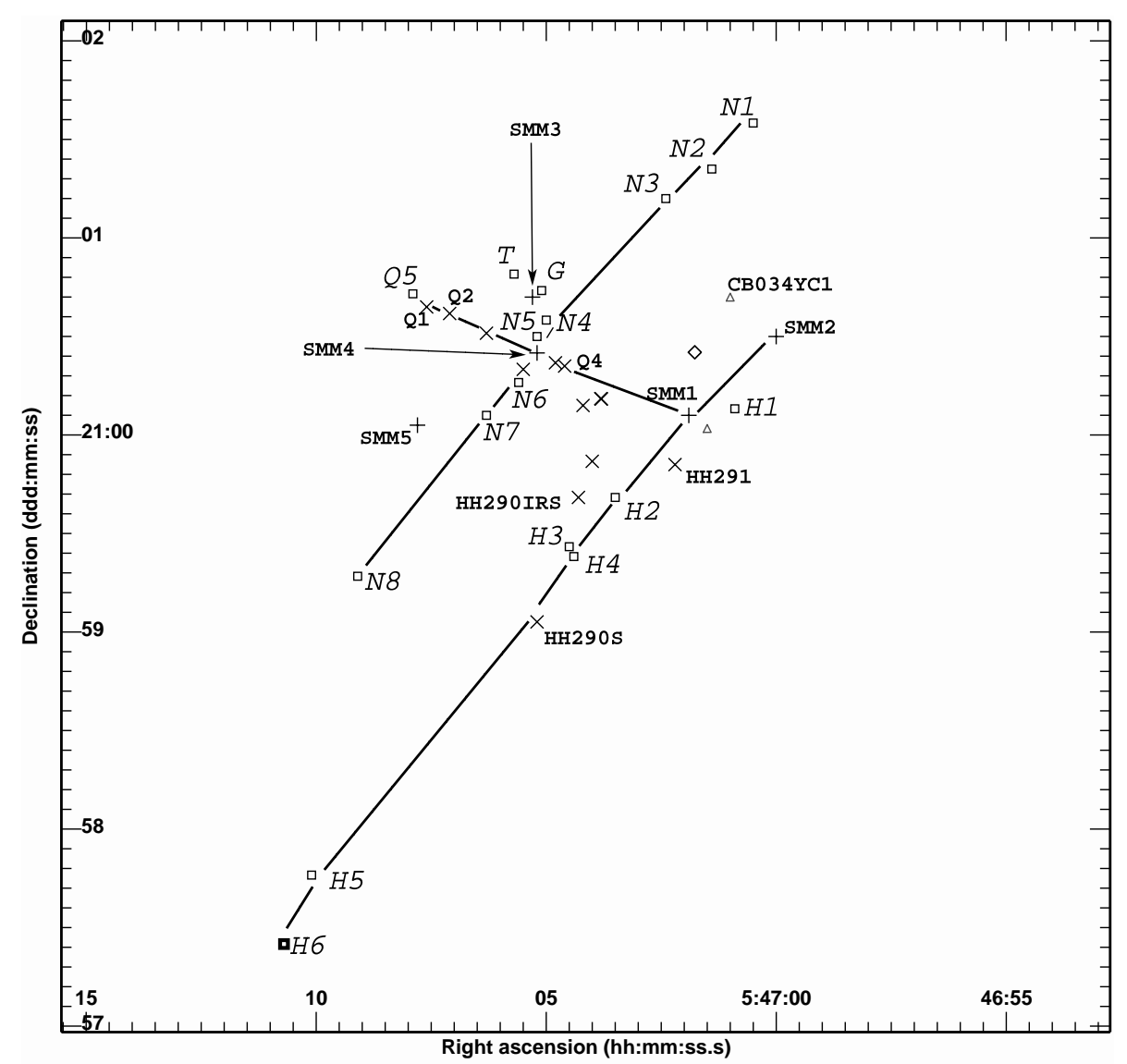

Fig. 6. Schematic map of the $\mathrm{H}, \mathrm{N}$ and $\mathrm{Q}$ near-infrared $\mathrm{H}_{2}$ outflows in CB 34. The squares are $\mathrm{H}_{2}$ knots discovered here. All other symbols are the same as in Fig. 1.

the CO emission. Such a conclusion would apply to any region where there are multiple outflows.

We remark that the age estimated from the CO outflow would be sufficient for jets to penetrate out to many tens of parsecs, given a propagation speed of $40 \mathrm{kms}^{-1}$. This suggests that molecular flows reach a limiting size of 1-5 parsecs during the Class $0 / 1$ phase, and then shrink in apparent size as the largest scale structure becomes disconnected from the source and rapidly fades. Then, during the Class 2 phase the outflows are further reduced to of order 0.1 pc, as measured (Hogerheijde et al. 1998). Evidence is presented below that the observed infrared outflow is indeed of a similar size to the globule.

The new CO data displayed in Fig. 7 provide information on the cool molecular gas which has been disturbed or swept up by the protostellar outflows. Each map covers an interval of $1 \mathrm{~km} \mathrm{~s}^{-1}$ around the inner $2^{\prime}$. The overall structure of the high velocity lobes is very similar to the maps presented by Yun \& Clemens (1994) in the ${ }^{12} \mathrm{CO} J=1-0$ line. However, the south-western lobe of high velocity gas (which is visible both in redshifted and blueshifted emission at relatively low velocities) displays a clear bipolar morphology at higher velocities, with a redshifted lobe extending to the south-east and a blueshifted lobe to the north-west of the position of SMM1. This bipolar flow seems to be fairly well collimated. Regarding the high-velocity gas in the north-eastern part of the map, mainly red-shifted emission is detected with this emission showing considerable substructure. Blue-shifted emission is not or only marginally detected; better data might be useful to further constrain the presence and distribution of high-velocity gas in the north-eastern part of the cloud.

\subsection{Individual $\mathrm{H}_{2}$ structures}

As opposed to aligned knots, a true jet-type structure N6 is detected within the SMM4 core (Fig. 15). The compact knot located at the northern edge of N6 suggests that the source of the $\mathrm{N}$ jet is located within this core. In fact, the $\mathrm{Q}$ and $\mathrm{N}$ jets appear to cross at the position of SMM4.

We note that other knots are extended in the direction of the N6 jet, including H5 (Fig. 6). The extension of H5 towards $\mathrm{H} 6$ is evidence that these outer knots, aligned with the inner knots $\mathrm{H} 1-\mathrm{H} 4$, are indeed part of the $\mathrm{H}$ outflow rather than a chance conjunction. Knot H6 (Fig. 16a) is at the end of the observed $\mathrm{H}$ jet but does not possess a bow or arc structure. It would appear to be a structure within the supposed jet or along a cavity wall, with the jet actually extending beyond H6, out of the globule, although no further infrared structure south of the globule edge is found (see Figs. 16a, 14d and 1). Similarly, the knots extending to the north of the $\mathrm{N}$ jet possess no bow 


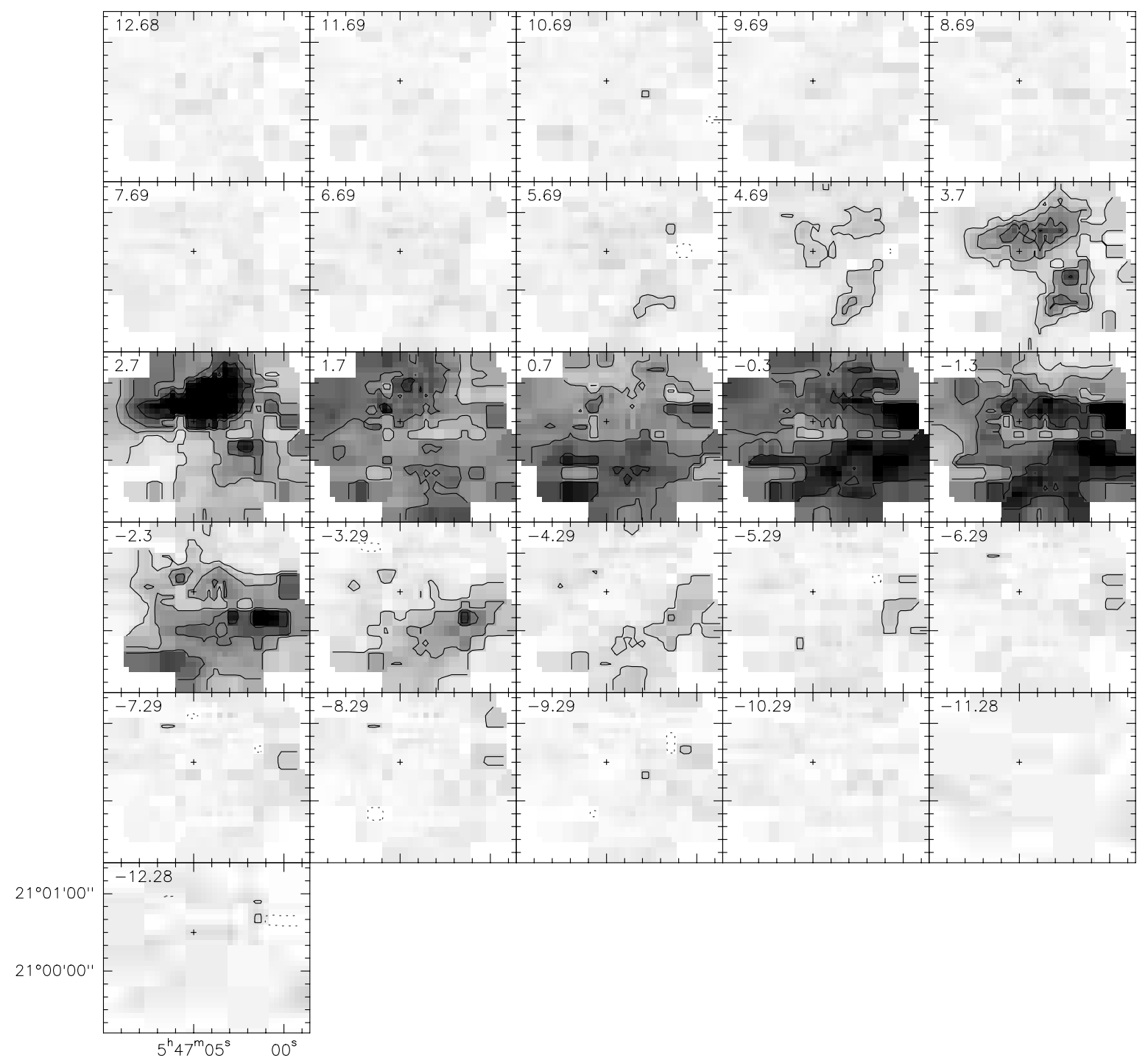

Fig. 7. ${ }^{12} \mathrm{CO}(2-1)$ velocity channel maps of $\mathrm{CB} 34$. The data have been resampled from the original velocity resolution of $0.42 \mathrm{~km} \mathrm{~s}^{-1}$ to $1.0 \mathrm{~km} \mathrm{~s}^{-1}$. The central velocity of each channel is indicated. The pixel size is $4^{\prime \prime}$, the beam size at $230 \mathrm{GHz}$ is about $11^{\prime \prime}$ (note however that only a $40 \times 40^{\prime \prime}$ area centered at the small cross has spatially fully sampled data; in the remaining area, the data are undersampled).

or cometary appearance, again suggesting that this jet has also exited from the globule.

These structures suggest an explanation for the strong asymmetry in the jet extensions from the probable sources. If the cloud were spheroidal with the $\mathrm{N}$ outflow near the rear surface, then N8 would demarcate the location where the jet exits from the rear surface of the globule, while N1 (Fig. 16b) would travel further through the globule to reach and exit on the near surface. On the other hand, if the SMM1 core is nearer to the front of the globule, then the receding southern $\mathrm{H}$ jet (as suggested by the CO lobes) could exit from near the middle section of the globule (see Fig. 1), producing a longer jet as it cuts its way through more globule material. The forward-moving northern part of the jet would then exit in a short length from the front of the cloud. Hence, the asymmetries can be produced by the source locations within the globule. Against this picture is the location of the optical knot HH $290 \mathrm{~S}$ which is located in the receding N-flow (see Fig. 6), thus deep within the globule. The optical extinction is 5-15 mag. for the embedded stars within the inner arcminute. HH $290 \mathrm{~S}$ is, however, $85^{\prime \prime}$ south of the central star E (Fig. 2, and lies in a region associated with less reddened stars (Fig. 2).

\subsection{The protostars and cores}

Matching protostars to outflows and jets is not unique without spectroscopic information or proper motions. Furthermore, at the distance of CB34, each submillimetre source could consist of a group of protostars, as explicitly demonstrated by Huard et al. (2000). 


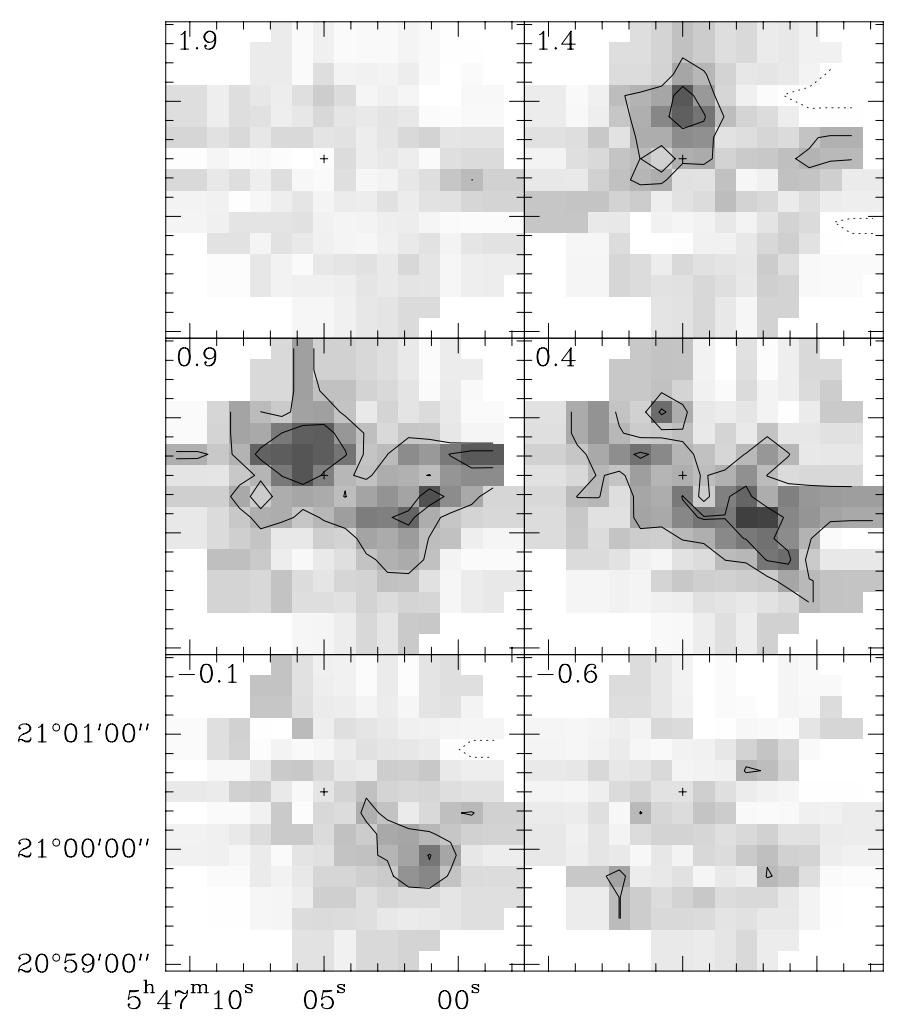

Fig. 8. $\mathrm{H}^{13} \mathrm{CO}^{+}$velocity channel maps of $\mathrm{CB} 34$. The pixel size is $11^{\prime \prime}$, the beam size at $86 \mathrm{GHz}$ is about $27^{\prime \prime} . \mathrm{H}^{13} \mathrm{CO}^{+}$is a tracer of high density gas. Two major condensations are seen, corresponding roughly to SMM1 to the south-west, centered at $v \sim 0.4 \mathrm{~km} \mathrm{~s}^{-1}$, and SMM4 to the north-east, centered at $v \sim 0.9 \mathrm{~km} \mathrm{~s}^{-1}$.

Protostars in the SMM1 core may be responsible for both the $\mathrm{H}$ and $\mathrm{Q}$ jets (Fig. 6). This core, of size $\sim 10^{\prime \prime}$, contains the IRAS source IRAS $0540+2059$ but was previously not associated with near-infrared emission. It is flattened at $450 \mu \mathrm{m}$ with a minor axis of $70^{\circ}$ (Huard et al. 2000), almost orthogonal to the Q-outflow of $68^{\circ}$.

In contrast, the large-scale globule, on scales of 12 arcmin, is flattened almost in the perpendicular direction to the core. This is evident in the HCN molecule (148 ${ }^{\circ}$, Afonso et al. 1998) and the far-infrared continuum (149 ${ }^{\circ}$, Huard et al. 2000). In comparison, the orientations of the $\mathrm{H}$ jet and $\mathrm{N}$ jet are both $\sim 150^{\circ}$. Hence, the major outflows are aligned with either their core or the globule minor axis. It is also interesting to note that the parallel outflows are also parallel to the galactic plane $\left(152^{\circ}\right)$. In contrast, the CO $J=2-1$ outflow from SMM1 has a rather badly defined orientation of $\sim 115-130^{\circ}$, which supports our assertion above that the CO lobes are produced by several outflows with a wide spread in ages while the $\mathrm{H}_{2}$ emission originates only from the latest outflows.

\subsection{The globule}

The extent of the globule is found from the photometric results (Fig. 4). Analysis of our wide field reveals that the the reddened stars (i.e. $H-K>1.0$ ) lie predominantly within a distance of $\sim 100^{\prime \prime}$ from the globule centre, (object $\mathrm{E}$ ). Slightly reddened stars $(1.0>H-K>0.7)$ extend out to $\sim 200^{\prime \prime}$. Furthermore, Fig. 4 demonstrates that the number of embedded stars $(H-K>0.7)$ per unit radius is roughly constant, with $18,19,17$ and 12 objects lying within the first four $50^{\prime \prime}$ radius intervals. Hence the observed number per unit surface area is inversely proportional to the radial distance. This indicates that the reddened stars are internal to the globule, not background.

The $200^{\prime \prime}$ radius halo of stars can be generated from a $40^{\prime \prime}$ radius core (i.e. the region containing SMM1 and SMM4) in $4 \times 10^{6} \mathrm{yr}$, if stars formed at a constant rate with an average radial expansion speed of $\sim 0.3 \mathrm{~km} \mathrm{~s}^{-1}$. This speed would appear plausible, given the relative motions observed in the core in the $\mathrm{H}^{13} \mathrm{CO}^{+}$line (Fig. 8). Note that there are fewer highly reddened stars to the south of object E, suggesting that the star formation has shifted to the north by a distance of $20^{\prime \prime}$ (Fig. 5). Clearly, stars have been forming for a long time within CB 34 and the centre of activity may have shifted northwards from HH 290IRS to the vicinity of object E, while more dense gas still remains further north.

The channel maps in $\mathrm{H}^{13} \mathrm{CO}^{+}$, a tracer of high density material in dense cores, demonstrate some evidence for large-scale rotation of $1.5 \mathrm{~km} \mathrm{~s}^{-1}$ over $90^{\prime \prime}$ (Fig. 8). With this interpretation, a mass of $\sim 90 /\left(\cos ^{2} \theta\right) M_{\odot}$ within a radius of $45^{\prime \prime}$ is derived, where $\theta$ is the inclination of the rotation axis to the line of sight. This is consistent with the mass derived from CS measurements of $170 M_{\odot}$ (Launhardt \& Henning 1998). SMM4 is redshifted by $0.5 \mathrm{~km} \mathrm{~s}^{-1}$ relative to SMM1. SMM2 is also identified in this molecule.

We also find complex internal velocity structure on the scale of $10^{\prime \prime}$, in the ${ }^{12} \mathrm{COJ}=1-0$ line (Fig. 7). This structure suggests that the inner $2^{\prime}$ of the globule is being stirred up by many outflows. The numerous $\mathrm{H}_{2}$ knots spread about the inner globule may thus be part of several cavity walls. The turbulent motions of $\Delta v \sim 1.0 \mathrm{kms}^{-1}$ combined with a total globule mass of $M_{\mathrm{c}} \sim 170 M_{\odot}$ and radius of $R_{\mathrm{c}}=0.75 \mathrm{pc}\left(100^{\prime \prime}\right)$, imply an energy decay rate of order $M_{\mathrm{c}}\left(\Delta v^{3} /\left(2 R_{\mathrm{c}}\right)=0.04 L_{\odot}\right.$ (Mac Low 1999). Therefore, quantitatively, the mechanical power of the outflows is sufficient to maintain the turbulence. The decay timescale from the largest scales is $R_{\mathrm{c}} / \Delta v=7.5 \times 10^{5} \mathrm{yr}$.

A further feature to note is the gap in CO emission at all velocities directly between SMM1 and SMM4, with stronger blueshifted emission to the south. This suggests that star formation, which first occured near HH290IRS, was later centered in this gap, dispersing the material and compressing the surrounding cores. This has now triggered a third phase of star formation in the adjacent cores. Note also the appearance of weak blue-shifted emission at all velocities in the range $-2-9 \mathrm{~km} \mathrm{~s}^{-1}$ from SMM2. This is the third most massive core in the globule, and may thus be just beginning to form a low-mass star as star formation gradually moves onwards in the north-west direction. 
Table 2. The diffuse halo brightness in mag/ $/ \operatorname{arcsec}^{2}$, found on inspection of large-scale regions not confused by point sources in individual $J, H$ and $K_{\mathrm{s}}$ images. Radial distances are from object E.

\begin{tabular}{lcccc}
\hline Name & Radius $\left({ }^{\prime \prime}\right)$ & $J$ & $H$ & $K_{\mathrm{s}}$ \\
\hline Extended halo & 65 & 21.0 & 19.8 & 18.7 \\
Main halo & 50 & 20.2 & 19.0 & 18.2 \\
Inner halo & 30 & 19.5 & 18.2 & 17.0 \\
\hline
\end{tabular}

CB 34 is not fully isolated. Its neighbour, CB 33, is at a projected distance of just $5 \mathrm{pc}$ and connects on to a wide ridge of material as shown in the IRAS HIRES ${ }^{4}$ image requested from IPAC ${ }^{5}$ (Fig. 9). Filamentary structures are also present between the two globules, with clumps within $\sim 2$ pc. For material which now forms CB 34 to have become disconnected from the ridge about $10^{6} \mathrm{yr}$ ago would require a separation speed of $2 \mathrm{~km} \mathrm{~s}^{-1}$, about equal to the gravitational escape speed from an average molecular cloud. This material would still, however, have to condense into the Bok globule.

\subsection{A diffuse near infrared halo}

We detect a weak halo of diffuse emission in the $J, H$ and $K_{\mathrm{s}}$ filters centered on the submillimetre and far infrared cores. Fig. 10 displays the diffuse halo in the $H$ band. Figure 11 presents the radially-averaged halo brightness for all three bands. The numerous locations where stars have been subtracted hamper the extraction of radial profiles. Furthermore, the halo consists of a largescale diffuse component plus numerous localised regions of scattered light from young stars within the globule, as discussed below.

We estimate a main halo of radius $50^{\prime \prime}(0.37 \mathrm{pc}$ at $1500 \mathrm{pc}$ ). An inner halo which covers just the submillimetre cores SMM1 and SMM4 is also defined in Table 2. An extended halo, down to the $K_{\mathrm{s}}$ band noise level, is of radius of $65^{\prime \prime}(0.48 \mathrm{pc})$. The extended halo has an intensity of $\sim 18.7 \mathrm{mag} / \operatorname{arcsec}^{2}$ in the $K_{\mathrm{s}}$ band (Table 2). This converts to $4 \times 10^{-4} \mathrm{erg} \mathrm{s}^{-1} \mathrm{~cm}^{-2} \mathrm{sr}^{-1}$. To determine the physical process producing the halo, we generated maps which locate the pixels which possess $H-K$ in specific ranges. Quite remarkably, for the range of values $(H-K)=0.5-0.9 \mathrm{mag}$, the halo closely silhouettes the dense far-infrared cores mapped by Huard et al. (2000), shown here in Figs. 12 and 13.

${ }^{4}$ HIRES uses the Maximum Correlation Method (MCM, Aumann et al. 1990) to produce images with better than the nominal resolution of the Infrared Astronomical Satellite (IRAS) data.

5 IPAC is Infrared Processing and Analysis Center established as the NASA archival center for the IRAS data and operated by the JPL and California Institute of Technology (Caltech) http://www.ipac.caltech.edu
The halo is not a result of incomplete sky subtraction. Firstly, the halo region was well within all the frames taken. Secondly, the halo extends far beyond the core where a number of small halos around bright stars could combine to produce weak general diffuse emission. Thirdly, the $J-H$ and $H-K$ colours of the diffuse halo are distinct from the embedded stars.

Magnitudes for the diffuse halo, estimated directly from contour maps in the three bands, are given in Table 2. These values are not consistent with thermal $\mathrm{H}_{2}$ emission from shocks, which predicts $(H-K)=1.5-3.4 \mathrm{mag}$ (Smith 1995), far too red.

Scattering from an extended distribution of reddened stars within the globule would appear relevant to specific locations but the observed diffuse halo is generally weaker in the $H$ band, the halo possessing $H-K=0.8-1.2$ and $J-H=1.2-1.3$. These values are, therefore, also not consistent with unreddened or reddened main-sequence stellar photospheres. We also expect that CB 34 is shielded from the UV photons of the ambient interstellar radiation field.

Scattering of radiation from the deeply embedded infrared-excess stars could produce the halo. These stars are redder than the halo $(J-H$ exceeding 1.7$)$, which would be consistent with the stronger scattering of shorter wavelength radiation although the extra path length of this radiation would tend to have the opposite effect, reddening the emission. We find 23 embedded protostars with estimated luminosities of $10 L_{\odot}$ within a core radius of $65^{\prime \prime}$. In comparison, the total bolometric luminosity of the cold dust in the submillimetre cores is $\sim 240 L_{\odot}$, consistent with the stellar content. Therefore, given about two magnitudes of $K$-band extinction, it is clear that the infrared radiation from the protostars is largely absorbed and re-radiated by the cold dust with an average intensity of $\sim 2.5 \times 10^{-3} \mathrm{erg} \mathrm{s}^{-1} \mathrm{~cm}^{-2} \mathrm{sr}^{-1}$. The fraction of scattered near-infrared radiation which escapes the cloud depends on several factors, including the clumpiness and albedo. With two magnitudes of extinction and an effective albedo of 0.25 , the halo intensity level would be $\sim 1 \times 10^{-4} \mathrm{erg} \mathrm{s}^{-1} \mathrm{~cm}^{-2} \mathrm{sr}^{-1}$. This is an estimate for the total scattered light, and is a factor of 4 lower than the observed $K$-band halo. This tentatively suggests, given the uncertainties involved, that scattered protostellar light is a minor contribution to the diffuse halo.

The $H-K$ and $J-H$ colours correspond to that of reddened $\mathrm{H}_{2}$ fluorescence or any other mechanism which produces a cascade down the rotational and vibrational energy ladders of $\mathrm{H}_{2}$. The inner halo displays more reddening, thus accounting for the anticorrelation of low $H-K$ with the dust maps. We have calculated the fluorescent colours from the line fluxes measured for NGC 2023 (Burton et al. 1998) and predicted from detailed calculations (Black \& van Dishoeck 1987). These are: $H-K=$ $0.5, J-H=0.6$ and $H-K=0.7, J-H=0.5$, respectively. These colours are indeed consistent with stronger $H$-band emission than from stellar photospheres, as found for the diffuse halo. Fluoresence implies a halo reddening corresponding to $\Delta(J-H) \sim 0.6-0.8$. This is consistent 


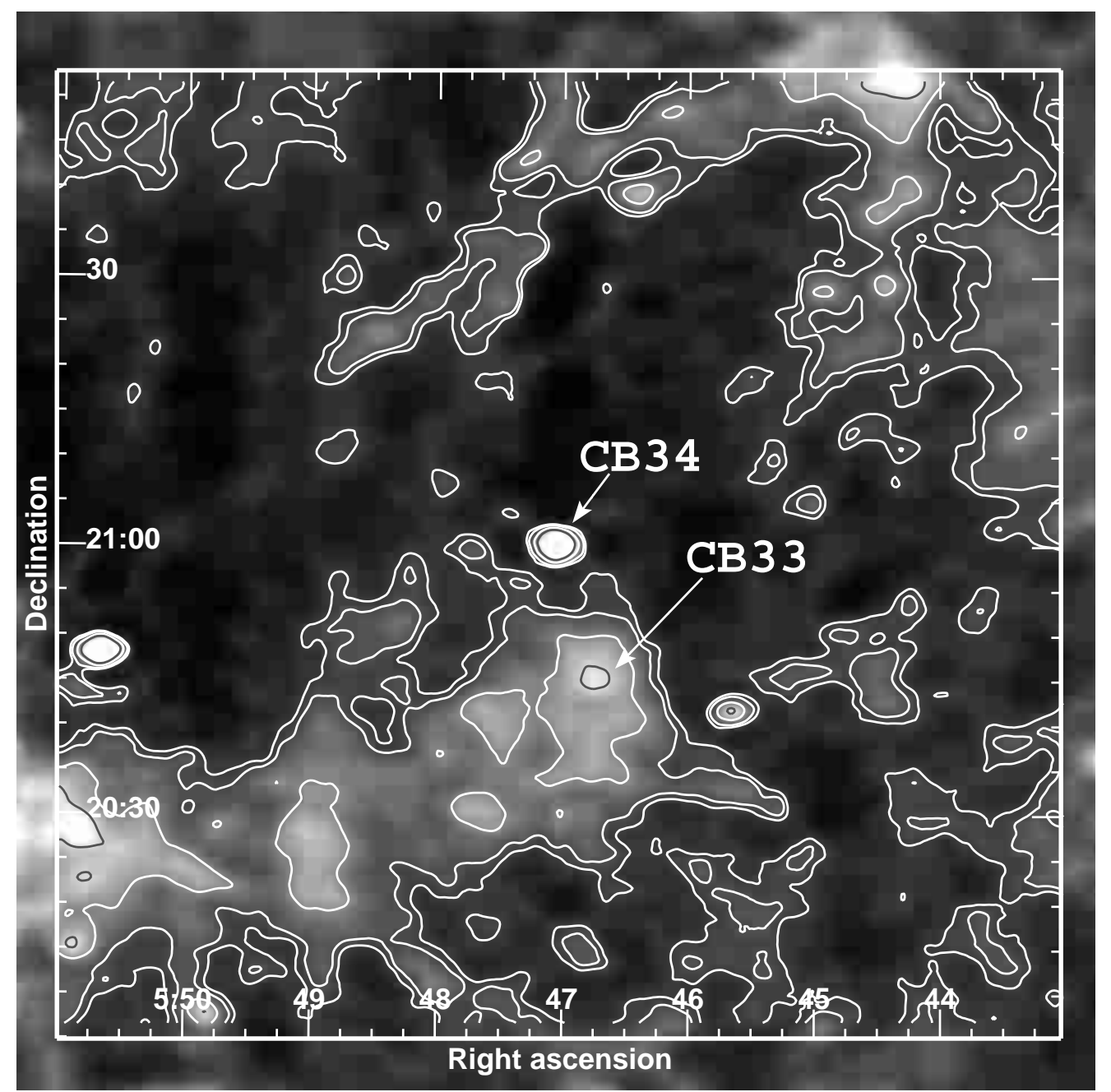

Fig. 9. IRAS HIRES $100 \mu \mathrm{m}$ map of the CB 34 region after 20 iterations. Image size is $2^{\circ} \times 2^{\circ}$. The displayed contour levels are $0.1,1,2,4$ and 8 Jy. Courtesy of the IPAC CALTECH http://www.ipac.caltech.edu.

with the reddening of the stars projected onto the diffuse halo of $\Delta(J-H) \sim 0.5-0.9$, despite the general difference in absolute $J-H$ values.

\subsection{Origin of the diffuse halo}

We now discuss the various physical processes which could produce diffuse near-infrared $\mathrm{H}_{2}$ emission: $\mathrm{H}_{2}$-formation on grains; impact excitation of $\mathrm{H}_{2}$ by secondary electrons produced by the absorption of $\mathrm{X}$-rays from embedded young objects; impact excitation of electronic states of $\mathrm{H}_{2}$ and direct impact excitation of vibrational levels in the $\mathrm{H}_{2}$ electronic ground state by secondary electrons produced by cosmic-ray ionisations; fluorescent excitation of $\mathrm{H}_{2}$ by internal UV photons emitted from embedded young stars.

Interstellar molecular hydrogen is formed on the surface of grains (Hollenbach \& Salpeter 1971; Jura 1975). Duley \& Williams (1993) calculated the total $\mathrm{H}_{2}$ flux for canonical cloud parameters. For their assumptions, including steady state cloud parameters, formation rate of $10^{-17} / \mathrm{n} \mathrm{s}^{-1}$ and five per cent of the reformations leading to $1-0 \mathrm{~S}(1)$ photons, their calculation yields a $1-0 \mathrm{~S}(1)$ intensity of just $10^{-8} \mathrm{erg} \mathrm{cm}^{-3} \mathrm{~s}^{-1}$, for a cloud of mean depth 0.6 pc. This is far below detectable limits. Duley \& Williams noted, however, that mixing between gas phases would raise the atomic density from the steady state value of $n=1 \mathrm{~cm}^{-3}$ to $100 \mathrm{~cm}^{-3}$, increasing the $1-0 \mathrm{~S}(1)$ intensity to $10^{-6} \mathrm{erg} \mathrm{cm}^{-3} \mathrm{~s}^{-1}$. This is still well below present measurement capabilities.

Le Bourlot et al. (1995) calculated the resulting near-infrared emission spectra of $\mathrm{H}_{2}$ leaving the grains. The authors presented $H$ and $K$ band spectra assuming a) equipartition between translational, ro-vibrational, and vibrational degrees of freedom, b) $\mathrm{H}_{2}$ formation in the highest vibrational level, and c) $\mathrm{H}_{2}$ formation in $v^{\prime \prime}=6$ but at a low rotational excitation temperature of $65 \mathrm{~K}$. The latter is suggested from catalysis experiments (Duley \& Williams 1986). They found total $K$-band fluxes below $10^{-7} \mathrm{erg} \mathrm{cm}^{-3} \mathrm{~s}^{-1}$, which confirm the above conclusion.

Molecule formation, on the other hand, would provide a full explanation for the measured diffuse halo. In a formation scenario, as opposed to reformation, the entire cloud halo forms dynamically on the same time scale 


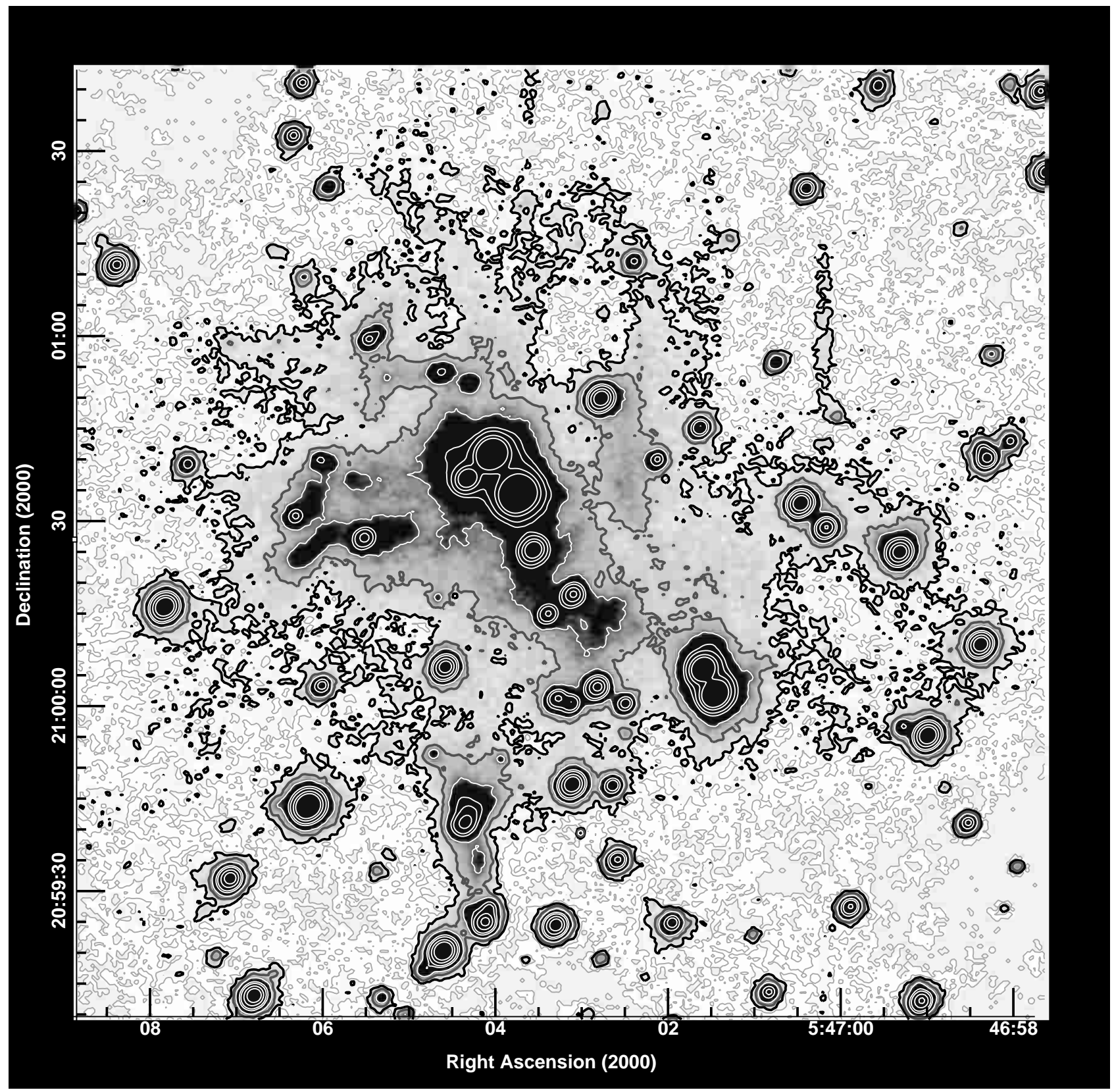

Fig. 10. Near infrared $H$-band image of CB 34 with the Calar Alto $3.5 \mathrm{~m}$ and the Omega Prime camera. The three lowest contour levels, corresponding to the values in Table 2, are 19.8, 19.0 and $18.2 \mathrm{mag} / \mathrm{arcsec}^{2}$.

as molecule formation. Hence the atomic density is high because equilibrium has not been reached. This scenario will be explored separately (Smith et al. 2001). Here, we note that with an atomic density of $10^{4} \mathrm{~cm}^{-3}$ in the halo, the predicted 1-0 $\mathrm{S}(1)$ intensity is $10^{-4} \mathrm{erg} \mathrm{cm}^{-3} \mathrm{~s}^{-1}$. The integrated $K \mathrm{~s}$ intensity is then predicted to be $\sim 10^{-3} \mathrm{erg} \mathrm{cm}^{-3} \mathrm{~s}^{-1}$, close to that observed. Smith et al. (2001) demonstrate more generally that molecule and cloud formation can both occur on time scales of $10^{6} \mathrm{yr}$, much shorter than expected in many previous models, yet consistent with Bok globule statistics. This is due to the revision in our ideas of the interstellar medium away from the concept of pressure-matched phases to that of supersonic turbulence.
In a gas of low fractional ionisation, the emission from $v^{\prime \prime}=0,1,2$ is dominated by direct impact excitation from non-thermal secondary electrons produced by cosmic ray absorptions in dark clouds (Gredel \& Dalgarno 1995). Cosmic ray ionisations are always present, and provide in fact the main ionisation source which drives the chemistry in the dense molecular parts. The resulting $\mathrm{H}_{2}$ emission spectrum, no matter what is assumed for the $\mathrm{H}_{2}$ formation process, is extremely red, with most of the emission occurring in the $K$-band (see e.g. Figs. $3 \mathrm{a}-\mathrm{d}$ of Le Bourlot et al. 1995). Colours are dominated by values of $(H-K) \gg 1 \mathrm{mag}$, which is in conflict with the observations presented above. 


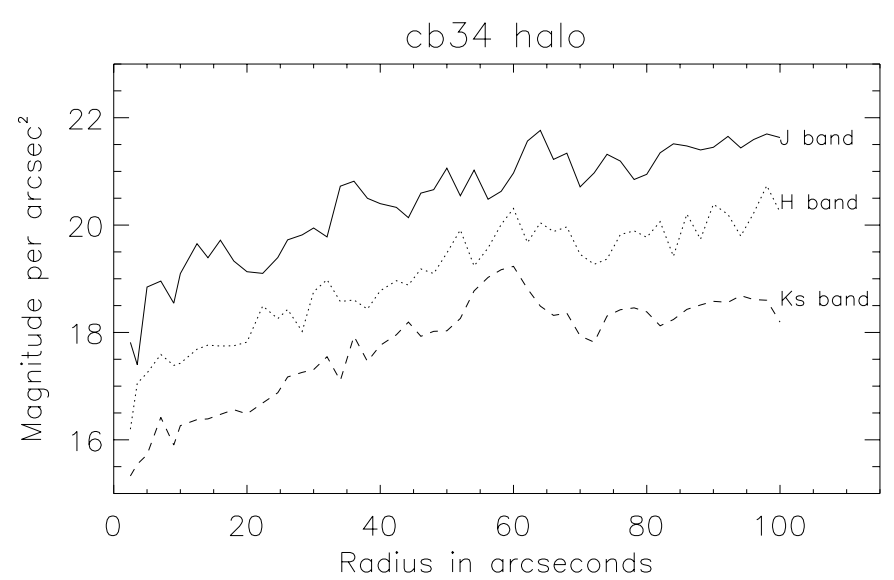

Fig. 11. The brightness in the globule (per square arcsecond) averaged over each $2.5^{\prime \prime}$ for all three NIR bands after extraction of the point sources. The radial distance is measured from source E (cf. Figs. 4 and 5). The background values are reached at a radius of $\sim 65^{\prime \prime}$ for $J$ and $K_{\mathrm{s}}$ and $80^{\prime \prime}$ for the $H$-band.

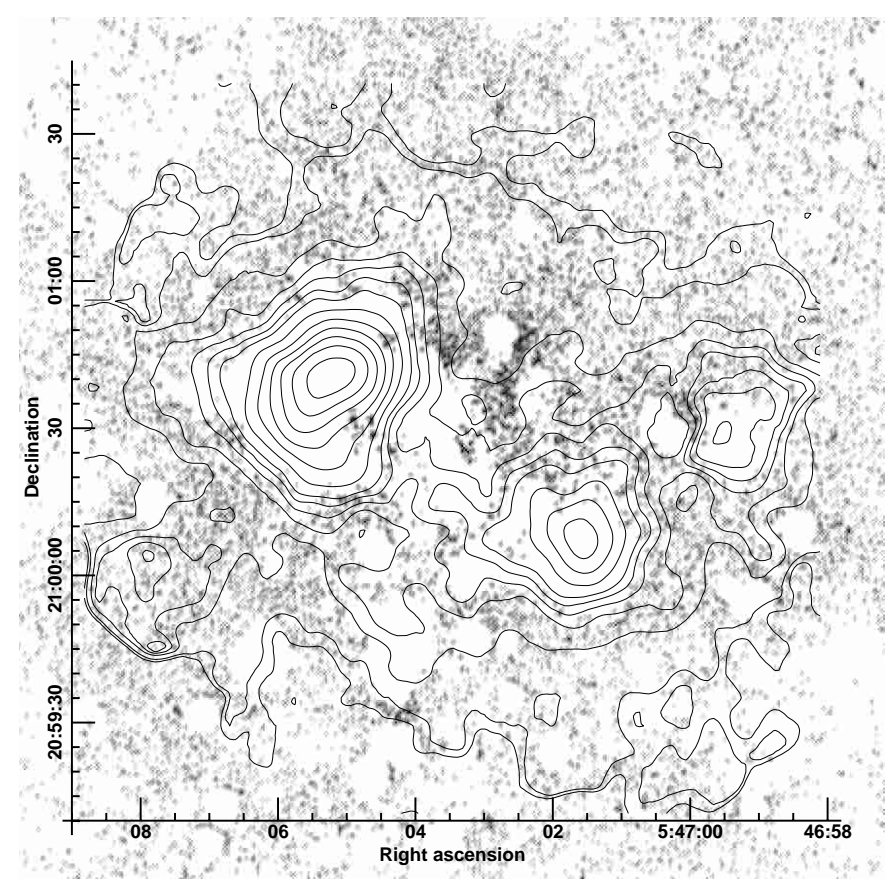

Fig. 12. The $850 \mu \mathrm{m}$ contour map, kindly provided by Huard et al. (2000), overlaid on the greyscale surface brightness map of the locations associated only with the $H-K$ colour in the range $0.5-0.9$. This is the range of values corresponding to cascade $\mathrm{H}_{2}$ mechanisms with little or no reddening. Note that there is a quite detailed, although not perfect, anti-correlation.

Apart from penetrating cosmic rays, absorptions of $\mathrm{X}$-rays in dense molecular material resulting from young objects embedded in CB 34 may produce non-thermal, secondary electrons as well. The response of $\mathrm{H}_{2}$ to $\mathrm{X}$-rays was calculated by Gredel \& Dalgarno (1995) and Tiné et al. (1997). Again, the preferential excitation of the $v^{\prime \prime}=1,2$ levels by electron impact results in high intensities of the transitions out of these levels. Consequently, the expected near-infrared emission from X-ray absorptions is very red,

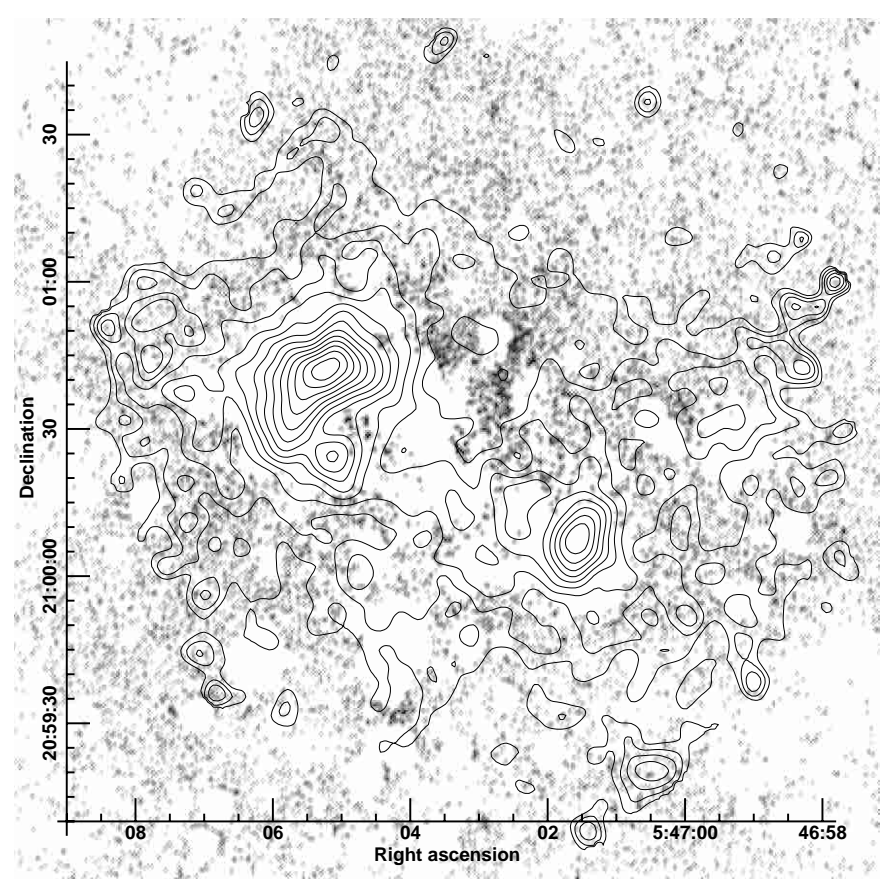

Fig. 13. The $450 \mu \mathrm{m}$ contour map of Huard et al. (2000) overlaid on the greyscale image of the locations associated with the $H-K$ colour in the range $0.5-0.9$.

with $(H-K) \gg 1 \mathrm{mag}$. This is also in conflict with the observed colour of the halo in CB 34.

The near-infrared emission spectrum expected from embedded sources of UV photons is significantly different from that expected from X-rays. Opposite to the direct excitation of ro-vibrational levels in all electronic states of $\mathrm{H}_{2}$, including its ground state, UV photons populate electronic states allowed by the selection rules of electric dipole radiation only. The fluorescence process mainly in the Lyman and Werner bands populates the ro-vibrational levels of the ground state which is followed by cascading. (Black \& van Dishoeck 1987). The expected color of the pure fluorescence process is $(H-K)=0.5-0.7 \mathrm{mag}$. UV fluorescent excitation, however, requires the presence of massive stars. Even NGC 2023, the brightest source of fluorescent $\mathrm{H}_{2}$, powered by a B star generates a 1-0 S(1) intensity of $7 \times 10^{-5} \mathrm{erg} \mathrm{s}^{-1} \mathrm{~cm}^{-2} \mathrm{sr}^{-1}$. Here, however, we have no such massive star.

We conclude that fast molecule formation within an interstellar medium which is constantly evolving provides the most plausible explanation for the diffuse halo. This scenario will be explored elsewhere (Smith et al. 2001). Tests for the model could be based on the low $\mathrm{H}_{2} / \mathrm{CO}$ abundance, the effects of reformation heating and the $\mathrm{H}_{2}$ cascade spectrum. Near-infrared polarimetry would test the extent of the halo as a reflection nebula with internal sources of illumination.

\subsection{Origin of $C B 34$}

How did the globule form? If the diffuse halo is identified with hydrogen molecules, they form on the same time 


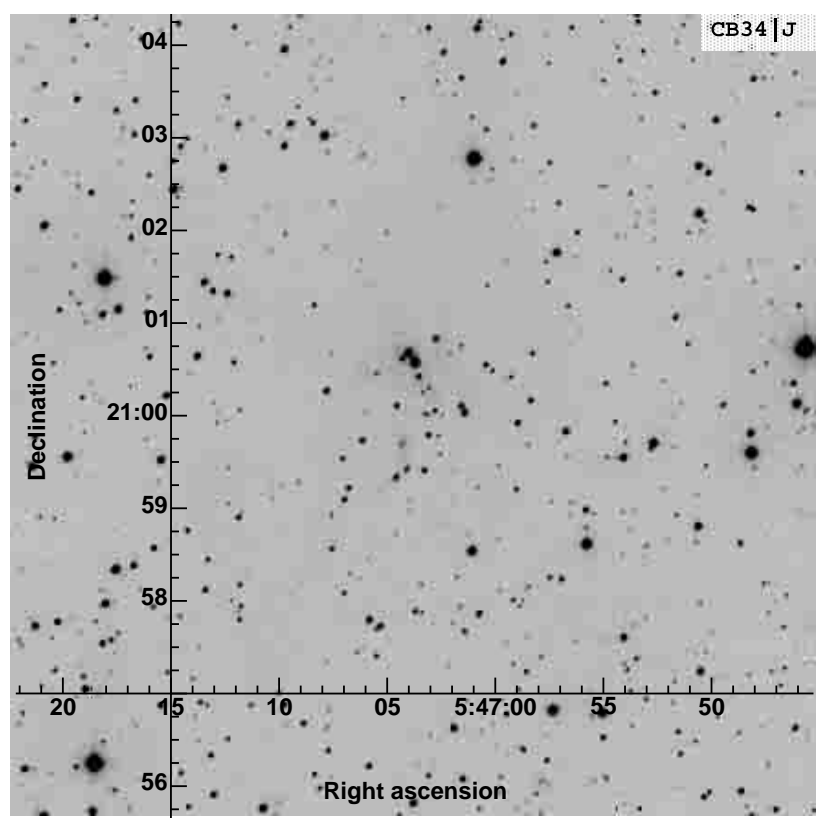

(a)

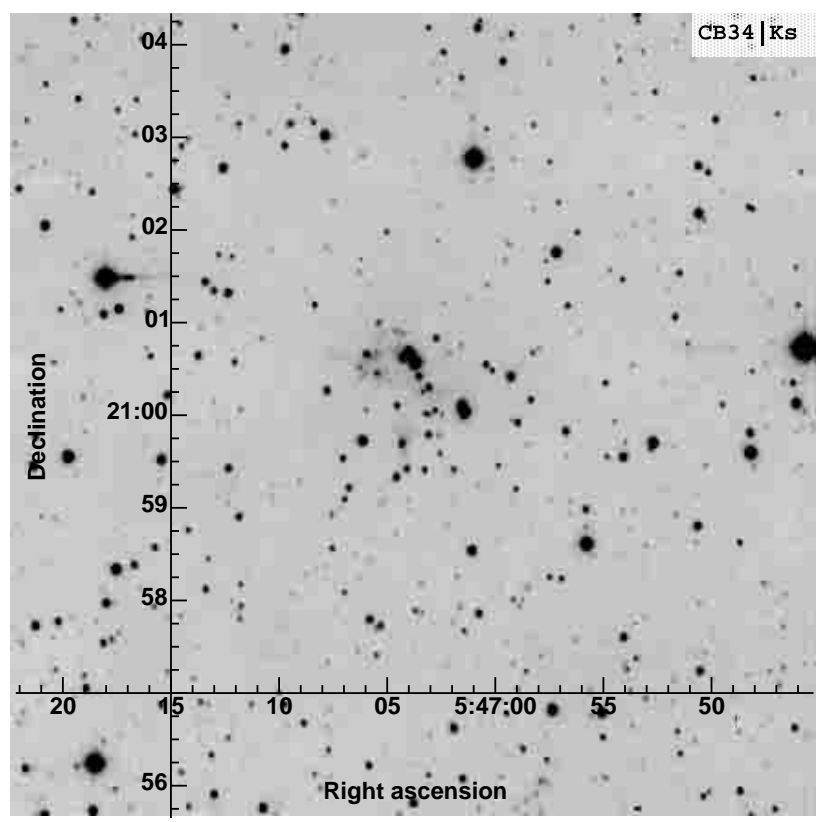

(c)

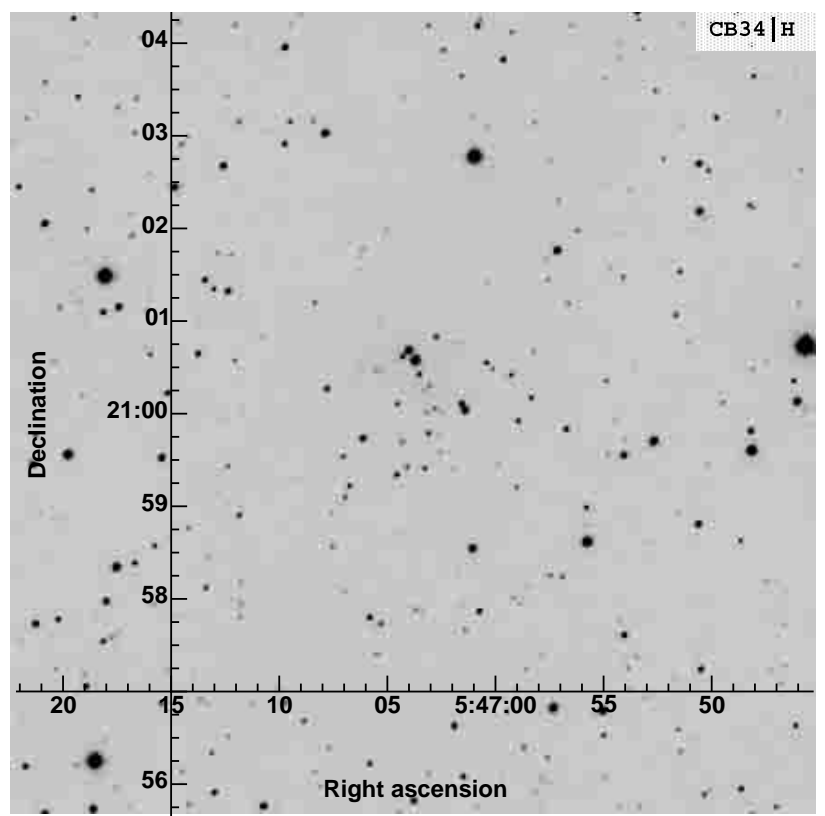

(b)

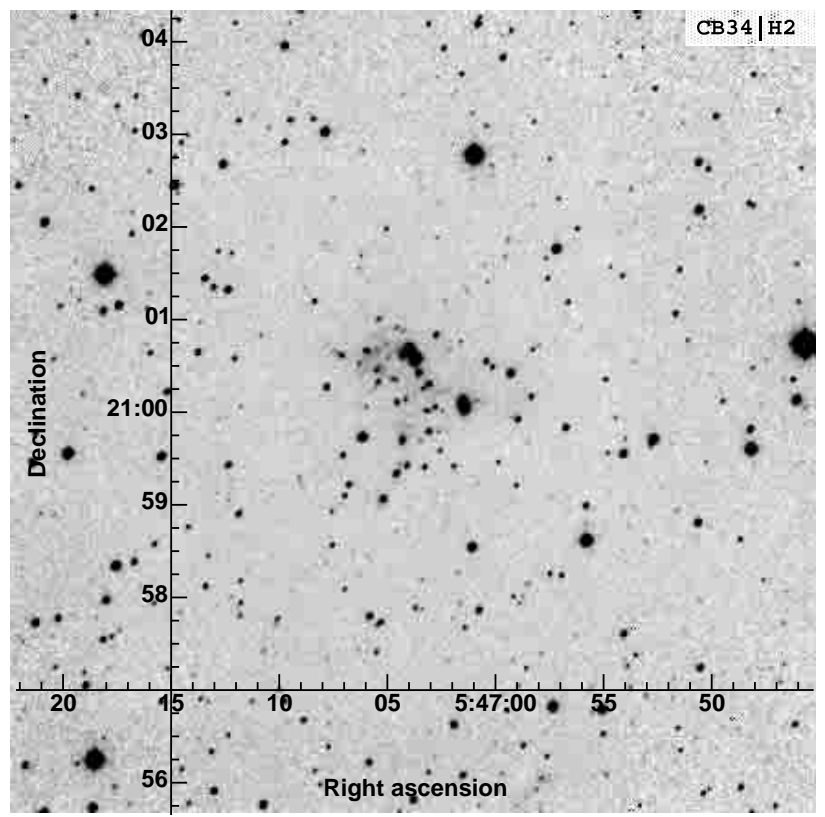

(d)

Fig. 14. Mosaics in $\left.J(\mathbf{a})), H(\mathbf{b})), K_{\mathrm{s}}(\mathbf{c})\right)$ and $\left.H_{2}(\mathbf{d})\right): X$-axes are Right Ascension and $Y$-axes are Declination (J2000). Images have been obtained after reducing and mosaicing data by DIMSUM package under IRAF. Sizes of images are $8.78 \times 8.75 \operatorname{arcmin}^{2}$.

scale of $10^{6} \mathrm{yr}$ as the globule age. This favours a model in which the cloud has formed by cooling and collapse of an atomic cloud. This cloud would have originally been, perhaps, a few parsecs in size and $10-100 \mathrm{~cm}^{-3}$ in density. Cloud formation is, however, a continuous process and the interpretation of the halo in terms of molecule formation suggests that the cloud is still growing in mass.
The original warm atomic cloud may have been associated with the end of a ridge of gas seen in the IRAS $100 \mu \mathrm{m}$ map. An alternative is that the cloud was torn off from a distant molecular cloud at the edge of the Gemini OB1 association. This would, however, imply an age of at least $10^{7} \mathrm{yr}$ which does not agree with other estimates. We thus conclude that CB34 has formed, along with 


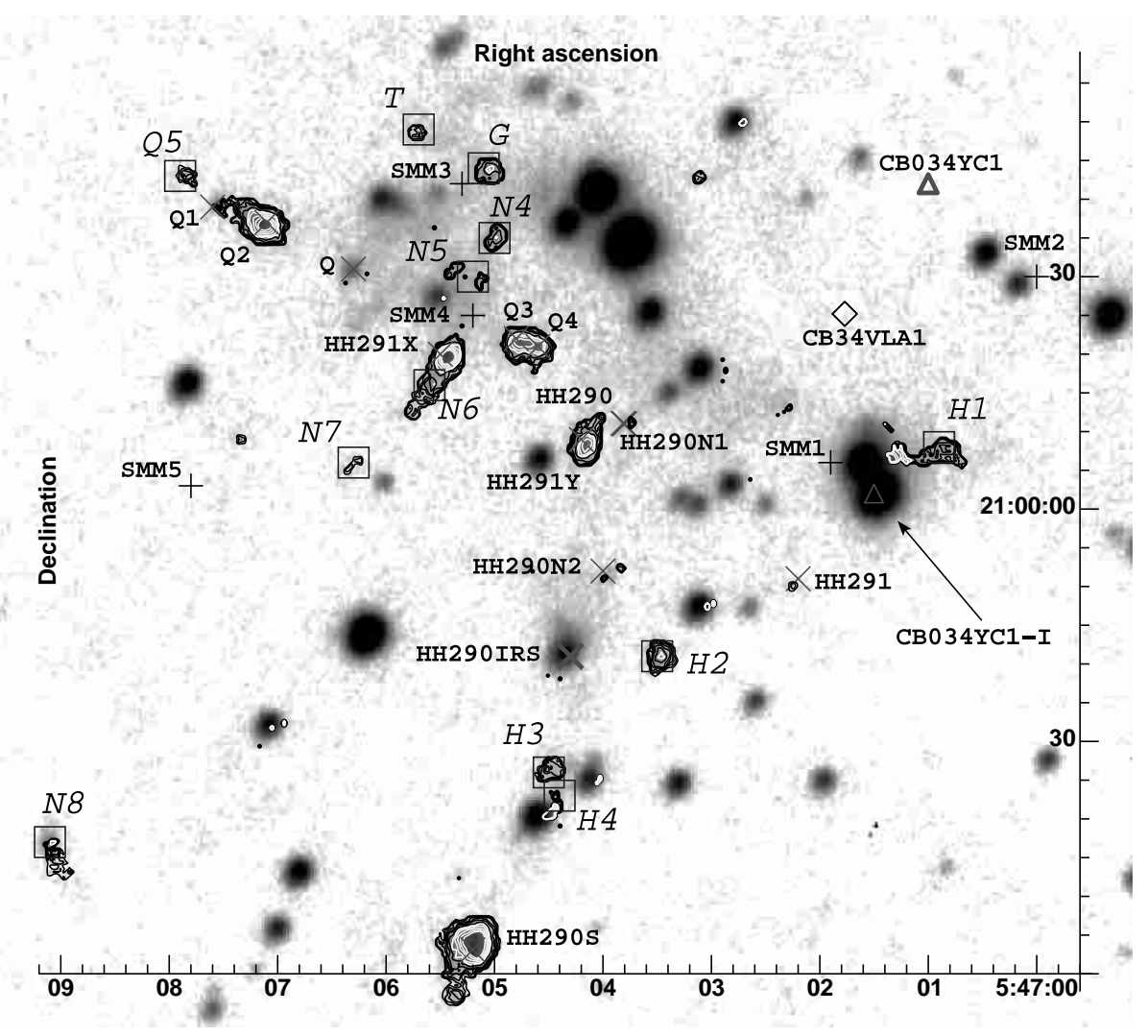

Fig. 15. The central part of CB 34 as described in Fig. 1.
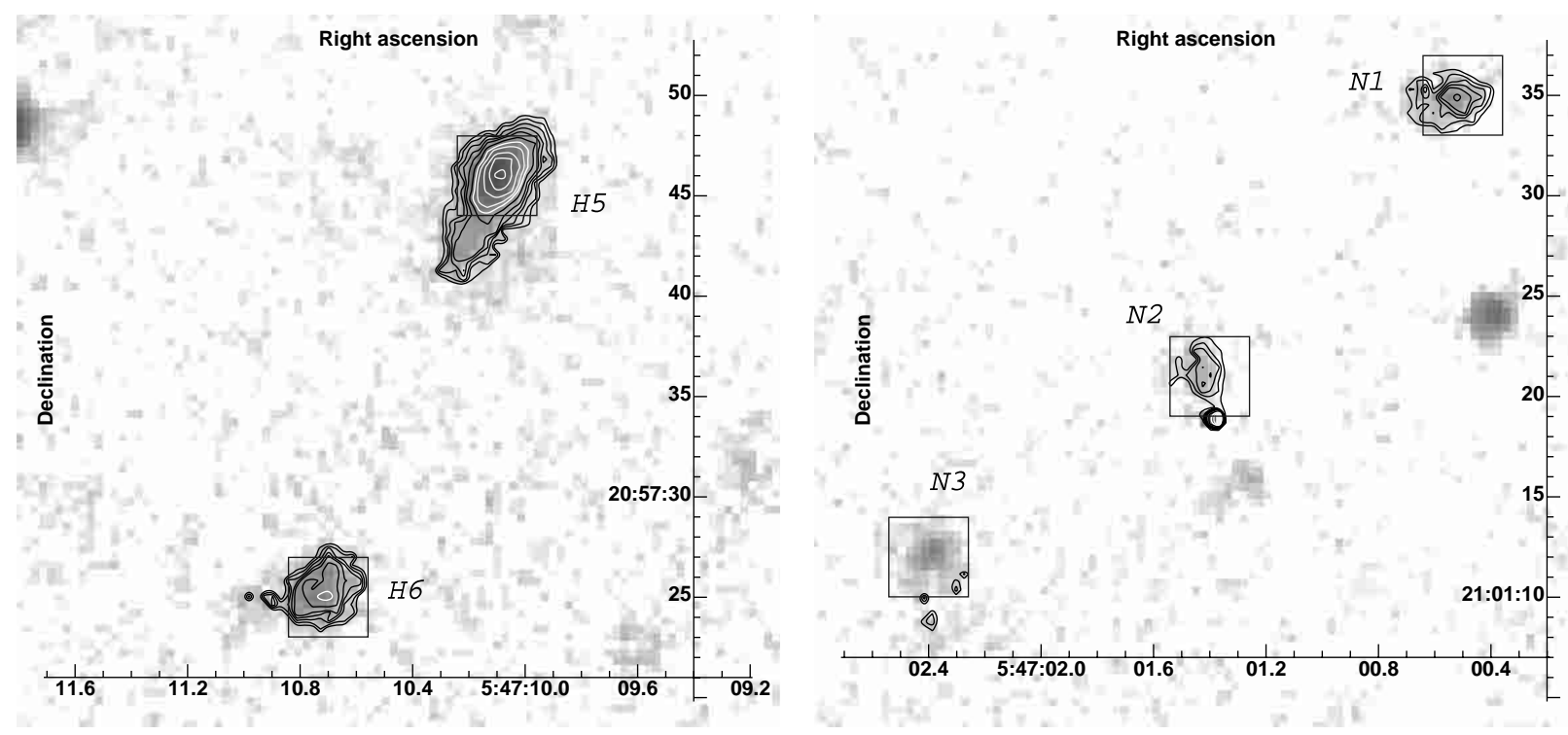

Fig. 16. Close-up views of the $\mathrm{H}_{2}$ objects in the continuum-subtracted 1-0 S(1) filter. a) Details of H5 and H6 knots; b) details of N1, N2 and N3 knots.

CB 33, near the end of a dust ridge. Triggering by compression may have instigated the collapse and shortened the molecule formation time scale. A triggering agent is, however, not evident and slow spontaneous formation, followed by the present rapid evolution, cannot be excluded.
How has the globule evolved? One possibility is that a roundish globule has undergone an inside-out collapse. This formed a central flattened core about $10^{6} \mathrm{yr}$ ago. Collapse proceeded fast $40^{\prime \prime}$ south of this core, between SMM1 and SMM4, and these protostars evacuated a 
central cavity in the flattened core. Turbulent motions, driven by the outflows, of $1-2 \mathrm{~km} \mathrm{~s}^{-1}$ led to members of the young group expanding to their present locations, up to $1 \mathrm{pc}$ from the original core. The protostellar winds have driven into the annulus, forming a core SMM1 on the front side and a core SMM4 towards the rear. This has led to predominantly red-shifted and blue-shifted molecular $\mathrm{H}^{13} \mathrm{CO}^{+}$lines, respectively, with accelerated material further away from the initial core. This may also explain the main $\mathrm{CO}$ bipolar outflow, which appears to be aligned along the cores rather than along the $\mathrm{H}_{2}$ flows (Fig. 7), with only a weaker blue lobe extending along the $\mathrm{N}$ outflow. The second phase of star formation begun about $5 \times 10^{5} \mathrm{yr}$ ago, within the two cores which have (in this scenario) a common rotation axis, which is ultimately responsible for generating parallel jets.

\section{Summary}

We have obtained and analysed wide-field near-infrared images of the Bok Globule CB 34 in the JHK continua and the $\mathrm{H}_{2} 2.12 \mu \mathrm{m}$, which reveal some of the properties of the cloud mass, distribution of young stars and their jets and shocked outflows. We have also obtained velocity channel maps in molecular lines, $\mathrm{CO}$ maps revealing the cool outflows and turbulent motions, and $\mathrm{H}^{13} \mathrm{CO}^{+}$maps displaying the dense cores.

Two new long extended infrared jets were discovered. These jets are detected over $1.2 \mathrm{pc}$ and $1.6 \mathrm{pc}$ to the outskirts of the globule. Since no terminating bow shock is found and the tracing $\mathrm{H}_{2}$ knots are confined to the dark cloud, it is proposed that the jets extend much further into a lower density halo. The two jets are extremely well collimated and are parallel to within $3^{\circ}$.

We estimate that the total shocked luminosity is comparable to the mechanical luminosity, derived from $\mathrm{CO}$ measurement, consistent with jet-driven non-evolving outflow structure. With an estimated dynamical age of $7.6 \times 10^{5}$ years, the mechanical luminosity and shocked luminosities are both $L_{\text {mech }}=0.02 L_{\odot}$. These outflows may be driven by low-mass very young stars. We note, however, that a more viable explanation is that the $\mathrm{CO}$ outflows are dominated by the accumulated outflowing mass from older outflows whereas the $\mathrm{H}_{2}$ is from shocks generated by the latest generation of outflows.

$J H K$ photometry yields the presence of a central concentration of reddened stars, and a surrounding lower density region of less reddened stars within the globule. The spatial spread suggests that the stars have been generated for $\sim 10^{6} \mathrm{yr}$ within the cores. The presence of a pre-mainsequence star, CB34FU (see Fig. 1), with an age of $\sim 10^{6} \mathrm{yr}$ supports this as a minimum age (Alves et al. 1997).

The two outflows appear to be driven from the two densest submillimetre cores which we detect here in the $\mathrm{H}^{13} \mathrm{CO}^{+}$molecule, a tracer of dense gas. The axis separating these cores is roughly transverse to the outflow direction.
A diffuse halo is detected in $J, H$ and $K$-band images, at a level consistent with that associated with the rapid formation of molecular hydrogen at a density of $10^{4} \mathrm{~cm}^{-3}$ in a time of order $10^{6} \mathrm{yr}$. This suggests that the globule has formed directly out of an atomic cloud associated with a dust ridge, from which it continues to accrete mass. The alternative remains that the diffuse halo is purely scattered light from infrared-excess protostars. There is, however, a clear observed deficit in the number of protostars as well as the dust bolometric luminosity, which makes this interpretation problematic.

Two phases of star formation are suggested. The first phase generated the wide distribution of young stars. This activity triggered further core formation in the north of the globule, leading to the present generation of dense cores, outflows and protostars.

Acknowledgements. We would like to thank Tracy Huard for supplying the far infrared images. We are extremely grateful to Martin Murphy for his support in establishing the data reduction software.

\section{References}

Adams, F. C., Lada, C. J., \& Shu, F. H. 1987, ApJ, 312, 788

Afonso, J. M., Yun, J. L., \& Clemens, D. P. 1998, AJ, 115, 1111

Alves, J. F., \& Yun, J. L. 1995, ApJ, 438, L107

Alves, J. F., Hartmann, L., Brieño, C., \& Lada, C. J. 1997, AJ, 113, 1395

Alves, J. F., Lada, C. J., \& Lada, E. 2001, in preparation

Aumann, H. H., Fowler, J. W., \& Melnyk, M. 1990, AJ, 99, 1674

Bizenberger, P., McCaughrean, M. J., Birk, C., Thompson, D., \& Storz, C. 1998, Proc. SPIE, 3354, 825

Black, J. H., \& van Dishoeck, E. F. 1987, ApJ, 322, 412

Bok, B. J., \& Reilly, E. F. 1947, ApJ, 105, 255

Burton, M. G., Howe, J. E., Geballe, T. R., \& Brand, P. W. J. L. 1998, PASA, 15, 194

Carpenter, J. M., Snell, R. L., \& Schloerb, F. P. 1995, ApJ, 445,246

Clemens, D. P., \& Barvanis, R. E. 1988, ApJS, 68, 257

Clemens, D. P., Yun, J. L., \& Heyer, M. H. 1991, ApJS, 75, 877

Cowie, L. L., Gardner, J. P., Lilly, S. J., \& McLean, I. S. 1990, ApJ, 360, L1

Duley, W. W., \& Williams, D. A. 1986, MNRAS, 223, 177

Duley, W. W., \& Williams, D. A. 1993, MNRAS, 260, 37

Eisenhardt, P., Dickinson, M., Stanford, S. A., Ward, J., \& Valdes, F. 1996, May, available from ftp>iraf .noao.edu/iraf/contrib/dimsumV2/

Gredel, R., \& Dalgarno, A. 1995, ApJ, 446, 852

Hogerheijde, M. R., van Dishoeck, E. F., Blake, G. A., \& van Langevelde, H. J. 1998, ApJ, 502, 315

Hollenbach, D., \& Salpeter, E. E. 1971, ApJ, 163, 155

Huard, T. L., Weintraub, D. A., \& Sendell, G. 2000, A\&A, 362, 635

Jura, M. 1975, ApJ, 197, 575 
Koornneef, J. 1983, A\&A, 128, 84

Lada, C. J., \& Adams, F. C. 1992, ApJ, 393, 278

Launhardt, R., Evans II, N. J., \& Wang, Y. 1998, ApJS, 119, 59

Le Bourlot, J., Pineau des Forêts, G., Roueff, E., Dalgarno, A., \& Gredel, R. 1995, ApJ, 449, 178

Launhardt, R., \& Henning, Th. 1997, A\&A, 326, 329

Mac Low, M.-M. 1999, ApJ, 524, 169

Moreira, M. C., \& Yun, J. L. 1995, ApJ, 454, 850

Rosen, A., \& Smith, M. D. 2001, in preparation

Scappini, F., Cecchi-Pestellini, C., Olberg, M., Casolari, A., \& Fanti, C. 1998, ApJ, 504, 866

Schilke, P., Walmsley, C. M., Pineau des Forêts, G., \& Flower, D. R. 1997, A\&A, 321, 293

Smith, M. D. 1991, MNRAS, 253, 175

Smith, M. D. 1995, A\&A, 296, 789
Smith, M. D. 1996, in Disks and Outflows around Young Stars, ed. S. Beckwith, et al. (Springer Heidelberg), CD-ROM, 809-815

Smith, M. D. 2000, IAJ, 27, 25

Stanke, T. 2000, Ph.D. Thesis, Univ. Potsdam

Stetson, P. B. 1987, PASP, 99, 191

Strom, K. M., Strom, S. E., Edwards, S., Cabrit, S., \& Skrutskie, M. F. 1989, AJ, 97, 1451

Tiné, S., Lepp, S., Gredel, R., \& Dalgarno, A. 1997, ApJ, 481, 282

Yun, J. L., \& Clemens, D. P. 1994, ApJ, 365, L73

Yun, J. L., \& Clemens, D. P. 1994, ApJS, 92, 145

Yun, J. L., Moreira, M. C., Torrelles, J. M., Afonso, J. M., \& Santos, N. C. 1996, AJ, 111, 841

Yun, J. L., Moreira, M. C., Alves, J. F., \& Storm, J. 1997, A\&A, 320, 167 